\title{
Tight-binding description of the quasiparticle dispersion of graphite and few-layer graphene
}

\author{
A. Grüneis, ${ }^{1,2, *}$ C. Attaccalite,,${ }^{3,4}$ L. Wirtz,${ }^{4}$ H. Shiozawa, ${ }^{5}$ R. Saito, ${ }^{6}$ T. Pichler, ${ }^{1}$ and A. Rubio ${ }^{3}$ \\ ${ }^{1}$ Faculty of Physics, University of Vienna, Strudlhofgasse 4, 1090 Wien, Austria \\ ${ }^{2}$ IFW Dresden, P.O. Box 270116, D-01171 Dresden, Germany \\ ${ }^{3}$ Departamento de Fisica de Materiales, Donostia International Physics Center, Spain European Theoretical Spectroscopy Facility \\ (ETSF), E-20018 San Sebastian, Spain \\ ${ }^{4}$ Institute for Electronics, Microelectronics, and Nanotechnology (CNRS UMR 8520), Department ISEN, \\ 59652 Villeneuve d'Ascq, France \\ ${ }^{5}$ Advanced Technology Institute, University of Surrey, Guildford, GU2 7XH, United Kingdom \\ ${ }^{6}$ Department of Physics, Tohoku University, Aoba, Sendai, 980-8578, Japan
}

(Received 9 August 2008; revised manuscript received 13 October 2008; published 19 November 2008)

\begin{abstract}
A universal set of third-nearest-neighbor tight-binding (TB) parameters is presented for calculation of the quasiparticle $(\mathrm{QP})$ dispersion of $N$ stacked $s p^{2}$ graphene layers $(N=1 \ldots \infty)$ with $A B$ stacking sequence. The present TB parameters are fit to $a b$ initio calculations on the $G W$ level and are universal, allowing to describe the whole $\pi$ "experimental" band structure with one set of parameters. This is important for describing both low-energy electronic transport and high-energy optical properties of graphene layers. The QP bands are strongly renormalized by electron-electron interactions, which results in a $20 \%$ increase in the nearest-neighbor in-plane and out-of-plane TB parameters when compared to band structure from density-functional theory. With the new set of TB parameters we determine the Fermi surface and evaluate exciton energies, charge carrier plasmon frequencies, and the conductivities which are relevant for recent angle-resolved photoemission, optical, electron energy loss, and transport measurements. A comparision of these quantitities to experiments yields an excellent agreement. Furthermore we discuss the transition from few-layer graphene to graphite and a semimetal to metal transition in a TB framework.
\end{abstract}

DOI: 10.1103/PhysRevB.78.205425

PACS number(s): 73.22.-f, 71.20.Tx, 79.60.-i

\section{INTRODUCTION}

Recently mono- and few-layer graphene (FLG) in an $A B$ (or Bernal) stacking is made with high crystallinity by the following methods: epitaxial growth on $\mathrm{SiC},{ }^{1,2}$ chemical vapor deposition on $\mathrm{Ni}(111),{ }^{3}$ mechanical cleavage on $\mathrm{SiO}_{2}$ (Refs. 4 and 5), and exfoliation. ${ }^{6}$ Graphene is a novel twodimensional (2D) and metastable material, which has sparked interest from both basic science and application point of view. ${ }^{7}$ A monolayer of graphene allows one to treat basic questions of quantum mechanics such as Dirac fermions or the Klein paradox ${ }^{8}$ in a simple condensed-matter experiment. The existence of a tunable gap in a graphene bilayer was shown by angle-resolved photoemission (ARPES), ${ }^{9}$ which offers a possibility of using these materials as transistors in future nanoelectronic devices that can be lithographically patterned. ${ }^{10}$ Furthermore a graphene layer that is grown epitaxially on a $\mathrm{Ni}(111)$ surface is a perfect spin filter device ${ }^{11}$ that might find applications in organic spintronics.

It was shown recently by ARPES that the electronic structure of graphene ${ }^{12}$ and its three-dimensional (3D) parent material, graphite, ${ }^{13-15}$ is strongly renormalized by correlation effects. To date the best agreement between ARPES and $a b$ initio calculations is obtained for $G W$ (Greens function $G$ of the Coulomb interaction $W$ ) calculations of the quasiparticle (QP) dispersion. ${ }^{16-19}$ The band structure in the local-density approximation (LDA) is not in good agreement with the ARPES spectra because it does not include the long-range electron correlation effects. The self-energy correction of the Coulomb interaction to the bare energy-band structure is cru- cial for determining the transport and optical properties (excitons) and related condensed-matter phenomena. For graphite, a semimetal with a tiny Fermi surface, the number of free electrons to screen the Coulomb interaction is very low $\left(\sim 10^{19}\right.$ carriers $\left.\mathrm{cm}^{-3}\right)$, and thus the electron-electron correlation is a major contribution to the self-energy correction. ${ }^{13}$ Theoretically, the interacting QP dispersion is obtained by the $G W$ approximation. The $G W$ calculations are computationally expensive and, thus, only selected $k$ points have been calculated. ${ }^{13}$ Therefore a tight-binding (TB) Hamiltonian with a transferable set of TB parameters that reproduces the QP dispersion in $s p^{2}$ stacked graphene sheets is needed for analysis of ARPES, optical spectroscopies, and transport properties for pristine and doped graphite and FLGs. So far there are already several sets of TB parameters published for graphene, FLG, and graphite. For graphene a third-nearestneighbor (3NN) fit to LDA has been performed. ${ }^{20}$ Recently, however it has been shown by ARPES that the LDA underestimates the slope of the electronic bands and also the trigonal warping effect. ${ }^{13}$ For bilayer graphene the parameters of the Slonzcewski-Weiss-McClure (SWMC) Hamiltonian have been fitted to reproduce double resonance Raman data. ${ }^{21} \mathrm{~A}$ direct observation of the QP band structure is possible by ARPES, and a set of TB parameters has been fitted to the experimental ARPES data of graphene grown on $\mathrm{SiC}^{22}$ As a result they obtained a surprisingly large absolute value of the nearest-neighbor $\pi$ hopping parameter of $5.13 \mathrm{eV}^{22}$ This is in stark contrast to the fit to the LDA calculation, which gives only about half of this value. ${ }^{20}$ Another issue regarding the TB fit to the electronic bands of graphene on a SiC substrate is the substrate interaction, which is also responsible for a gap opening and a distortion of the bands when com- 
pared to an isolated graphene sheet..$^{23}$ Considering the wide range of values reported for the hopping parameters, a reliable and universal set of TB parameters is needed that can be used to calculate the QP dispersion of an arbitrary number of graphene layers. The band structure of FLGs has been calculated $^{24}$ using first-nearest-neighbor in-plane coupling, which provides the correct band structure close to $K$ point. However, as we will show in detail in this paper, the inclusion of $3 \mathrm{NN}$ is essential in describing the experimental band structure in the whole Brillouin zone (BZ). The inclusion of second-nearest-neighbor ( $2 \mathrm{NN})$ interactions is responsible for breaking of the electron-hole symmetry. ${ }^{25}$ Since the distance ratio to second- and to third-nearest neighbors is 1.73/ 2.0 , inclusion of $3 \mathrm{NN}$ is also essential for reproducing the $a b$ initio data in the whole 3D BZ. It has already been shown in Ref. 26 that these very interactions have considerable magnitude in graphene and must also be considered to reproduce first-principles data in graphene nanoribbons.

In this paper, we present a TB formulation of the $\pi$ LDA energy band and QP dispersions of $A B$ stacked FLG and graphite. We have previously compared both $G W$ and LDA calculations to ARPES experiments and proven that LDA underestimates the slope of the bands and trigonal warping. ${ }^{13}$ Here we list the TB fit parameters of the QP dispersion [tight-binding $G W(\mathrm{~TB}-G W)$ ] and the corresponding LDA dispersion [tight-binding local-density approximation (TBLDA)] and show that the in-plane and out-of-plane hopping parameters increase when going from LDA to $G W$.

This new and improved set of TB-GW parameters is used for direct comparision to experiments. The parameters are obtained from a fit to QP calculations in the $G W$ approximation. This set of TB parameters works in the whole 2D (3D) $\mathrm{BZ}$ of FLG (graphite) and is in agreement to a wide range of different experiments. In addition we fit the parameters of the popular SWMC Hamiltonian that is valid close to the $K H$ axis of graphite. The main application of the parameter set given in this work are planar and infinite $s p^{2}$ bonded carbon sheets. In addition, also the quasiparticle band structure of one-dimensional graphene nanoribbons and large diameter carbon nanotubes can be calculated with this set of parameters.

This paper is organized as follows: in Sec. II we develop the 3NN TB formulation for graphite and FLG, and in Sec. III the SWMC Hamiltonian is revised. In Sec. IV a new set of TB parameters for calculating the QP dispersion of stacked $s p^{2}$ carbon is given. In Sec. V we compare the graphite electron energy-band dispersion of the LDA to the QP $(G W)$ dispersion. In Sec. VI we use the TB- $G W$ Hamiltonian and calculate the doping-dependent Fermi surface of graphite and estimate effective masses and free charge carrier plasmon frequencies of pristine and doped graphite. In Sec. VII we show the calculated QP dispersions of FLGs. In Sec. VIII we discuss the present results and estimate the exciton binding energies, transport properties, and the low-energy plasmon frequencies. In Sec. IX the conclusions of this work are given. Finally, in the Appendix, the analytical forms of the Hamiltonians for FLG and for graphite are shown.
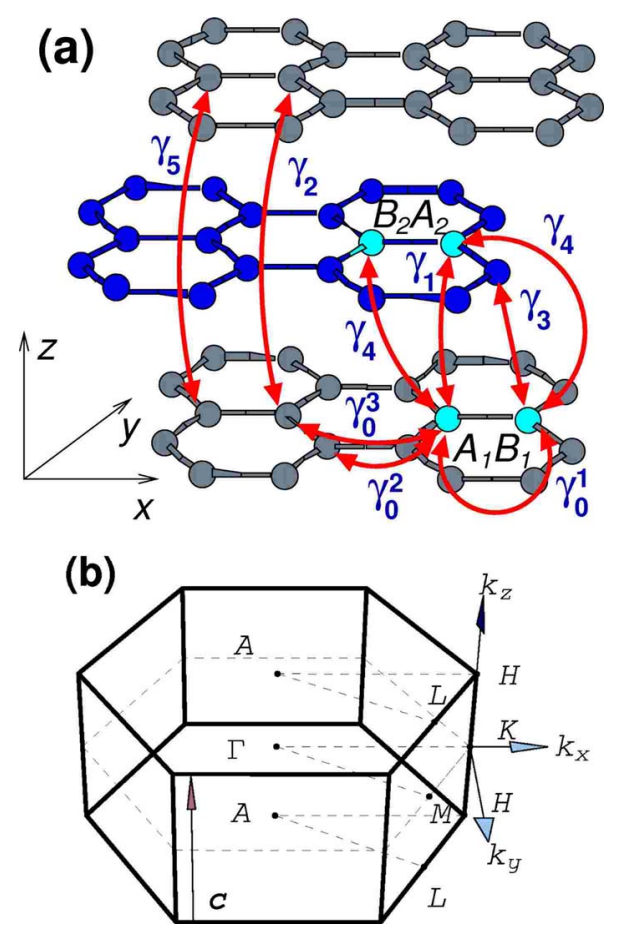

FIG. 1. (Color online) (a) The graphite unit cell consists of four atoms denoted by $A_{1}, B_{1}, A_{2}$, and $B_{2}$ (light blue). The red arrows denote the interatomic tight-binding hopping matrix elements $\gamma_{0}^{1}, \gamma_{0}^{2}, \gamma_{0}^{3}, \gamma_{1}, \ldots, \gamma_{5}$. The overlap matrix elements $s_{0}^{1}, s_{0}^{2}$, and $s_{0}^{3}$ (not shown) couple the same atoms as $\gamma_{0}^{1}, \gamma_{0}^{2}$, and $\gamma_{0}^{3}$. (b) The 3D Brillouin zone of graphite with the high-symmetry points and the coordinate system used throughout this work.

\section{THIRD-NEAREST-NEIGHBOR TIGHT-BINDING FORMULATION}

Natural graphite occurs mainly with $A B$ stacking order and has four atoms in the unit cell (two atoms for each graphene plane) as shown in Fig. 1(a). Each atom contributes one electron to the four $\pi$ electronic energy bands in the 3D BZ [see Fig. 1(b)]. FLG has $N$ parallel graphene planes stacked in an $A B$ fashion above one another; the unit cell of FLG is 2D, and the number of $\pi$ bands in the 2D BZ equals $2 N$. For graphite and FLG the TB calculations are carried out with a new 3NN Hamiltonian and in addition with the wellknown SWMC Hamiltonian ${ }^{27}$ that is valid in the vicinity to the Fermi level $\left(E_{F}\right)$. The TB parameters that enter these two Hamiltonians are $\gamma=\left(\gamma_{0}^{1}, \gamma_{0}^{2}, \gamma_{0}^{3}, s_{0}^{1}, s_{0}^{2}, s_{0}^{3}, \gamma_{1}, \ldots, \gamma_{5}, \Delta, E_{0}\right)$ for the 3NN Hamiltonian [shown in Fig. 1(a)] and $\gamma^{\prime}$ $=\left(\gamma_{0}^{\prime}, \ldots, \gamma_{5}^{\prime}, \Delta^{\prime}, E_{0}^{\prime}\right)$ for the SWMC Hamiltonian. The hopping matrix elements for the SWMC Hamiltonian are not shown here, but they have a similar meaning with the difference that only one nearest-neighbor in-plane coupling constant is considered (see e.g., Ref. 28 for an explanation of SWMC parameters). The hopping matrix elements for the 3NN Hamiltonian are shown in Fig. 1(a). The atoms in the 3D unit cell are labeled $A_{1}$ and $B_{1}$ for the first layer and $A_{2}$ and $B_{2}$ for the second layer. The $A_{2}$ atom lies directly above the $A_{1}$ atom in $z$ direction (perpendicular to the layers). Within the $x y$ plane the interactions are described by $\gamma_{0}^{1}$ (e.g., $A_{1} B_{1}$ and $\left.A_{2} B_{2}\right)$ for the nearest neighbors, and $\gamma_{0}^{2}$ and $\gamma_{0}^{3}$ for 
second-nearest and third-nearest neighbors, respectively. A further parameter, $\gamma_{1}\left(A_{1} A_{2}\right)$, is needed to couple the atoms directly above each other (in $z$ direction). The hoppings between adjacent layers of sites that do not lie directly above each other are described by $\gamma_{3}\left(B_{1} B_{2}\right)$ and $\gamma_{4}\left(A_{1} B_{2}\right.$ and $\left.B_{1} A_{2}\right)$. The small couplings of atoms in the next-nearest layer are $\gamma_{2}\left(B_{1} B_{1}\right.$ and $\left.B_{2} B_{2}\right)$ and $\gamma_{5}\left(A_{1} A_{1}\right.$ and $\left.A_{2} A_{2}\right)$.

The calculation shown here is valid for both graphite and FLGs with small adjustment as indicated when needed. The lattice vectors for graphite in the $x y$ plane are $\mathbf{a}_{1}$ and $\mathbf{a}_{2}$ and the out-of-plane lattice vector perpendicular to the layers is $\mathbf{a}_{3}$,

$$
\mathbf{a}_{1}=\left(\frac{\sqrt{3} a_{0}}{2},-\frac{a_{0}}{2}, 0\right), \quad \mathbf{a}_{2}=\left(\frac{\sqrt{3} a_{0}}{2}, \frac{a_{0}}{2}, 0\right), \quad \mathbf{a}_{3}=\left(0,0,2 c_{0}\right) .
$$

The C-C distance $a_{0}=1.42 \AA$ and the distance of two graphene layers $c_{0}=3.35 \AA$. For a FLG with $N$ layers and hence $2 N$ atoms only the $2 \mathrm{D}$ unit vectors $\mathbf{a}_{1}$ and $\mathbf{a}_{2}$. Similarly the electron wave vectors in graphite $\mathbf{k}=\left(k_{x}, k_{y}, k_{z}\right)$ have three components and in FLG $\mathbf{k}=\left(k_{x}, k_{y}\right)$. A TB method [or linear combination of atomic orbitals (LCAO)] is used to calculate the LDA and QP energy-band dispersion by two different sets of interatomic hopping matrix elements. The electronic eigenfunction $\Psi(\mathbf{r}, \mathbf{k})$ is made up from a linear combination of atomic $2 p_{z}$ orbitals $\phi(\mathbf{r})$, which form the $\pi$ electronic bands in the solid. The electron wave function for the band with index $J$ is given by

$$
\Psi^{J}(\mathbf{k}, \mathbf{r})=\sum_{s=A_{1}, B_{1}, \ldots, B_{N}} c_{s}^{J}(\mathbf{k}) \Phi_{s}(\mathbf{k}, \mathbf{r}),(J=1, \ldots, 2 N) .
$$

Here $J=1, \ldots, 2 N$ is the electronic energy-band index and $s$ in the sum is taken over all atomic $2 p_{z}$ orbitals from atoms $A_{1}, B_{1}, A_{2}, \ldots, B_{N}$. Note that for $3 \mathrm{D}$ graphite we have $N=2$. The $c_{s}^{J}(\mathbf{k})$ are wave-function coefficients for the Bloch functions $\Phi_{s}(\mathbf{k}, \mathbf{r})$. The Bloch wave functions are given by a sum over the atomic wave functions $\phi_{\text {s }}$ for each orbital in the unit cell with index $(\ell, m, m)$ multiplied by a phase factor. The Bloch function in graphite for the atom with index $s$ is given by

$$
\Phi_{s}(\mathbf{r}, \mathbf{k})=\frac{1}{\sqrt{U}} \sum_{\ell, m, n}^{U} \exp \left(-i \mathbf{R}_{s}^{\ell m n} \cdot \mathbf{k}\right) \phi_{s}\left(\mathbf{r}-\mathbf{R}_{s}^{\ell m n}\right)
$$

where $U$ is the number of unit cells and $\phi_{s}$ denotes atomic wave functions of orbital $s$. In graphite the atomic orbital $\phi_{s}$ in the unit cell with index $(\ell, m, m)$ is centered at $\mathbf{R}_{s}^{\ell m n}$ $=\ell \mathbf{a}_{1}+m \mathbf{a}_{2}+n \mathbf{a}_{3}+\mathbf{r}_{s}$ and in FLGs at $\mathbf{R}_{s}^{\ell m}=\ell \mathbf{a}_{1}+m \mathbf{a}_{2}+\mathbf{r}_{s}$.

For the case of FLGs $\Phi_{s}(\mathbf{r}, \mathbf{k})$ contains only the sum over the $2 \mathrm{D}$ in-plane $\mathbf{R}_{s}^{\ell m}$. The $2 N \times 2 N$ Hamiltonian matrix defined by $H_{s s^{\prime}}(\mathbf{k})=\left\langle\Phi_{s}(\mathbf{r}, \mathbf{k})|H(\mathbf{r})| \Phi_{s^{\prime}}(\mathbf{r}, \mathbf{k})\right\rangle$ and the overlap matrix is defined by $S_{s s^{\prime}}(\mathbf{k})=\left\langle\Phi_{s}(\mathbf{r}, \mathbf{k}) \mid \Phi_{s^{\prime}}(\mathbf{r}, \mathbf{k})\right\rangle$. For calculation of $H$ and $S$, up to third nearest-neighbor interactions (in the $x y$ plane) and both nearest- and next-nearest-neighbor planes (in $z$ direction) are included as shown in Fig. 1(a). The energy dispersion relations are given by the eigenvalues $E(\mathbf{k})$ and are calculated by solving

$$
H(\mathbf{k}) c(\mathbf{k})=S(\mathbf{k}) E(\mathbf{k}) c(\mathbf{k}) .
$$

In the Appendix, we show the explicit form of $H(\mathbf{k})$ for graphite and FLG with up to four layers.

\section{SWMC HAMILTONIAN}

The SWMC Hamiltonian has been extensively used in the literature. ${ }^{28}$ It considers only first-nearest-neighbor in-plane interaction and up to second-nearest-neighbor out-of-plane interaction and is valid close to the $K H$ axis of graphite. For small $k$ measured from the $K H$ axis (up to $0.15 \AA^{-1}$ ), both the $3 \mathrm{NN}$ and the SWMC Hamiltonians yield identical results. The eight TB parameters for the SWMC Hamiltonian were previously fitted to various optical and transport experiments. $^{28}$

For the cross sections of the electron and hole pockets analytical solutions have been obtained, and thus, it has been used to calculate the electronic transport properties of graphite. Thus, in order to provide a connection to many transport experiments from the past, we also fitted the LDA and $G W$ calculations to the SWMC Hamiltonian.

The TB parameters are directly related to the energy-band structure. For example, $\gamma_{0}^{\prime}$ is proportional to the Fermi velocity in the $k_{x} k_{y}$ plane, and $4 \gamma_{1}^{\prime}$ gives the bandwidth in the $k_{z}$ direction. The bandwidth of a weakly dispersive band in $k_{z}$ that crosses $E_{F}$ approximately halfway in between $K$ and $H$ is equal to $2 \gamma_{2}^{\prime}$, which is responsible for the semimetallic character of graphite. Its sign is of great importance for the location of the electron and hole pockets: a negative sign brings the electron pocket to $K$ while a positive sign brings the electron pocket to $H$. There has been positive signs of $\gamma_{2}^{\prime}$ reported earlier, ${ }^{29}$ but it has been found by Dresselhaus ${ }^{28,30}$ that the electron (hole) pockets are located at $K(H)$, which is in agreement with recent DFT calculations, ${ }^{13}$ tight-binding calculation, ${ }^{31}$ and experiments. ${ }^{28,32,13}$ The magnitude of $\gamma_{2}^{\prime}$ determines the overlap of electrons and holes and the volume of the Fermi surface. It, thus, also strongly affects the concentration of carriers and, hence, the conductivity and free charge carrier plasmon frequency. The effective masses for electrons and holes of the weakly dispersive energy band are denoted by $m_{z e}^{*}$ and $m_{z h}^{*}$, respectively. Their huge value also results from the small value of $\gamma_{2}^{\prime}$ and causes the low electrical conductivity and low plasmon frequency in the direction perpendicular to the graphene layers since $m_{z e}^{*}\left(\sqrt{m_{z e}^{*}}\right)$ enters the denominator in the expression for the Drude conductivity (free carrier plasmon frequency). The parameter $\gamma_{3}^{\prime}$ determines the strength of the trigonal warping effect $\left(\gamma_{3}^{\prime}\right.$ $=0$ gives isotropic equienergy contours) and $\gamma_{4}^{\prime}$ the asymmetry of the effective masses in valence band (VB) and conduction band $(\mathrm{CB})$. The other parameter from next-nearestneighbor coupling, $\gamma_{5}^{\prime}$, has less impact on the electronic structure: both the VB and $\mathrm{CB}$ at $K$ are shifted with respect to the Fermi level by $\Delta^{\prime}+\gamma_{5}^{\prime}$, causing a small asymmetry. ${ }^{28}$ Here $\Delta^{\prime}$ is the difference in the on-site potentials at sites $A_{1}\left(B_{2}\right)$ and $A_{2}\left(B_{1}\right) . \Delta^{\prime}$ is the value of the gap at the $H$ point. ${ }^{33}$ This crystal-field effect for nonzero $\Delta^{\prime}$ occurs in $A B$ stacked graphite and FLGs, but it does not occur in $A A$ stacked graphite and the graphene monolayer. The small on- 
TABLE I. 3NN tight-binding parameters for few-layer graphene and graphite. All values except the ones for $s_{0}^{1}$, $s_{0}^{2}$, and $s_{0}^{3}$ are in eV. The parameters of fits to LDA and $G W$ calculations are shown. The 3NN Hamiltonian is valid in the whole two (three) dimensional BZ of graphite (graphene layers).

\begin{tabular}{|c|c|c|c|c|c|c|c|c|c|c|c|c|c|}
\hline Method & $\gamma_{0}^{1}$ & $\gamma_{0}^{2}$ & $\gamma_{0}^{3}$ & $s_{0}^{1}$ & $s_{0}^{2}$ & $s_{0}^{3}$ & $\gamma_{1}$ & $\gamma_{2}$ & $\gamma_{3}$ & $\gamma_{4}$ & $\gamma_{5}$ & $E_{0}$ & $\Delta$ \\
\hline $3 \mathrm{NN}$ TB- $G W^{\mathrm{a}}$ & -3.4416 & -0.7544 & -0.4246 & 0.2671 & 0.0494 & 0.0345 & 0.3513 & -0.0105 & 0.2973 & 0.1954 & 0.0187 & -2.2624 & $0.0540^{\mathrm{b}}$ \\
\hline $3 N N$ TB-LDA ${ }^{a}$ & -3.0121 & -0.6346 & -0.3628 & 0.2499 & 0.0390 & 0.0322 & 0.3077 & -0.0077 & 0.2583 & 0.1735 & 0.0147 & -1.9037 & 0.0214 \\
\hline $\mathrm{EXP}^{\mathrm{c}}$ & -5.13 & 1.70 & -0.418 & -0.148 & -0.0948 & 0.0743 & & & & & & & \\
\hline $3 N N$ TB-LDA ${ }^{d}$ & -2.79 & -0.68 & -0.30 & 0.30 & 0.046 & 0.039 & & & & & & -2.03 & \\
\hline
\end{tabular}

aThis work.

${ }^{b}$ We adjusted the impurity doping level in order to reproduce the experimental value of $\Delta$.

${ }^{c}$ Fit to ARPES by Bostwick et al. (Ref. 22).

${ }^{\mathrm{d}}$ Fit to LDA by Reich et al. (Ref. 20).

site energy difference $\Delta^{\prime}$ appears in the diagonal elements of $H(\mathbf{k})$. It causes an opening of a gap at the $H$ point, which results in a breakdown of Dirac fermions in graphite and FLGs with $N>1$. Note that the magnitudes of this gap at $H$ depends sensitively on the doping level, ${ }^{34}$ which was adjusted in the first-principles calculations of this work in order to match the experimental values. This is reasonable when considering that the typical impurity concentrations are already sufficient to cause this small doping level. Finally, $E_{0}^{\prime}$ is set in such a way that the electronlike and holelike Fermi surfaces of graphite yield an equal number of free carriers. $E_{0}^{\prime}$ is measured from the bottom of the CB to the Fermi level.

\section{NUMERICAL FITTING PROCEDURE}

The $a b$ initio calculations of the electronic dispersion are performed on two levels: density-functional theory calculations (LDA) and QP dispersion calculations within the $G W$ approximation. We calculate the Kohn-Sham band structure within the LDA to DFT. ${ }^{35}$ Wave functions are expanded in plane waves with an energy cutoff at $25 \mathrm{Ha}$. Core electrons are accounted for by Trouiller-Martins pseudopotentials.

We then employ the $G_{0} W_{0}$ approximation using a plasmon-pole approximation for the screening ${ }^{16,36,37}$ to cal- culate the self-energy corrections to the LDA dispersion. For the calculation of the dielectric function $\epsilon(\omega, q)$ we use a $15 \times 15 \times 5$ Monkhorst-Pack $k$ sampling of the first BZ, and for conduction-band states with energies up to $100 \mathrm{eV}$ above the valence band ( 80 bands), calculations were performed using the code YAMBO. ${ }^{38}$ The details for the first-principles calculations are given elsewhere. ${ }^{34}$

For the fitting of the TB parameters to the ab initio (LDA and $G W$ ) calculations we used energies of the four $\pi$ bands of graphite at $\sim 100 k$ points. The points were distributed inside the whole 3D BZ of graphite. The fitting was performed with the 3NN Hamiltonian. In addition we chose a smaller subset of points inside a volume of $0.15 \times 0.15$ $\times 0.47 \AA^{-3}$ and fitted the SWMC parameters, which is frequently used in the literature. ${ }^{24,27,28}$

The set of TB parameters were fitted by employing a steepest-descent algorithm that minimizes the sum of squared differences between the TB and the $a b$ initio calculations. This involves solving Eq. (4) with different sets of TB parameters so as to approach a minimum deviation from the $a b$ initio calculations. Points close to $E_{F}$ were given additional weight so that the band crossing $E_{F}$ was described with a deviation less than $1 \mathrm{meV}$. This is important for an accurate description of the Fermi surface. In Table I we list the parameters for the $3 \mathrm{NN}$ Hamiltonian that can be used to

TABLE II. The SWMC tight-binding parameters for the LDA and the quasiparticle dispersion $(G W)$. All values are in $\mathrm{eV}$. These parameters are for the SWMC Hamiltonian (Refs. 24, 27, 28, and 39) which is valid close to the $K H$ axis.

\begin{tabular}{lllcccccc}
\hline \hline Method & \multicolumn{1}{c}{$\gamma_{0}^{\prime}$} & \multicolumn{1}{c}{$\gamma_{1}^{\prime}$} & \multicolumn{1}{c}{$\gamma_{2}^{\prime}$} & $\gamma_{3}^{\prime}$ & $\gamma_{4}^{\prime}$ & $\gamma_{5}^{\prime}$ & $E_{0}^{\prime}$ & $\Delta^{\prime}$ \\
\hline TB-GW a & 3.053 & 0.403 & -0.025 & 0.274 & 0.143 & 0.030 & -0.025 & $-0.005^{\mathrm{b}}$ \\
TB-LDA $^{\mathrm{a}}$ & 2.553 & 0.343 & -0.018 & 0.180 & 0.173 & 0.018 & -0.022 & -0.018 \\
EXP $^{\mathrm{c}}$ & 3.16 & 0.39 & -0.02 & 0.315 & 0.044 & 0.038 & -0.024 & -0.008 \\
LDA $^{\mathrm{d}}$ & 2.598 & 0.364 & -0.014 & 0.319 & 0.177 & 0.036 & -0.026 & -0.013 \\
EXP $^{\mathrm{e}}$ & 2.9 & 0.3 & & 0.1 & 0.12 & & & \\
KKR $^{\mathrm{f}}$ & 2.92 & 0.27 & -0.022 & 0.15 & 0.10 & 0.0063 & 0.0079 & -0.027 \\
\hline \hline
\end{tabular}

This work.

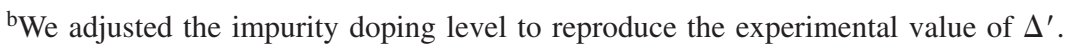

${ }^{c}$ Fit to experiment; see Dresselhaus et al. (Ref. 28).

${ }^{\mathrm{d}}$ Fit to LDA by Charlier et al. (Ref. 31).

eFit to double resonance Raman spectra by Malard et al. (Ref. 21).

${ }_{\mathrm{f}}^{\mathrm{F}}$ it to Korringa-Kohn-Rostocker first-principles calculation by Tatar and Rabi (Ref. 27). 


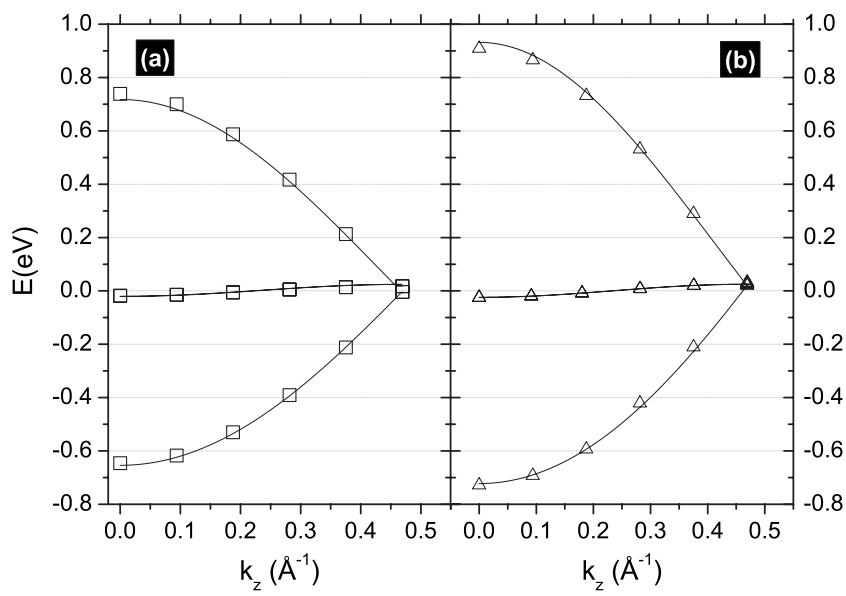

FIG. 2. The TB fit along $k_{z}$ direction at $k_{x}=k_{y}=0$ ( $K H$ axis) for (a) LDA and (b) $G W$. $\square$ denotes LDA calculations and $\triangle$ denotes $G W$ calculations taken from Ref. 13 that were used for the fitting.

calculate TB- $G W$ bands in the whole $3 \mathrm{D}$ BZ of graphite.

The parameters that were fit with the SWMC Hamiltonian are summarized in Table II. These TB parameters reproduce the LDA and the QP (fit to $G W$ ) calculated dispersions. Hereafter these fits are referred to as TB-LDA and TB- $G W$, respectively. We also list the values from other groups that were fit to experiments 28 and to LDA $^{31}$ and another firstprinciples calculation. ${ }^{27}$ It can be seen that the TB- $G W$ parameters for the nearest-neighbor coupling increase by about $20 \%$ when compared to TB-LDA. TB- $G W$ is also closer to the experimental TB parameters than the TB-LDA parameters. This indicates that electronic correlation effects play a crucial role in graphite and FLGs for interpreting and understanding experiments that probe the electronic energy-band structure.

\section{COMPARISION OF THE LDA TO THE QUASIPARTICLE ENERGY-BAND DISPERSION OF GRAPHITE}

We now compare the calculated TB-LDA to TB- $G W$, and we also show the result of the first-principles calculations that were used for fitting in order to illustrate the high quality of the fit. In Fig. 2 the full $k_{z}$ dispersion from $K$ to $H$ for (a) TB-LDA is compared to (b) TB- $G W$ calculations. It is clear that the bandwidth in $k_{z}$ increases by about $20 \%$ or $200 \mathrm{meV}$ when going from TB-LDA to TB- $G W$, i.e., when long-range electron-electron interaction is taken into account. Such an increase in bandwidth is reflected by the TB parameter $\gamma_{1}$ (in the SWMC model $4 \gamma_{1}^{\prime}$ is the total bandwidth in the out-ofplane direction). It can be seen that the conduction bandwidth increases even more than the valence bandwidth. The VB dispersion was measured directly by ARPES and gave a result in good agreement to the TB- $G W .{ }^{13}$

The dispersion parallel to the layers is investigated in Fig. 3 , where we show the $k_{y}$ dispersion for $k_{z}=0(K)$ and $k_{z}$ $=0.47 \AA^{-1}(H)$. Here the TB- $G W$ bands also become steeper by about $20 \%$ when compared to TB-LDA. This affects $\gamma_{0}^{1}$, $\gamma_{0}^{2}$, and $\gamma_{0}^{3}$, which are the in-plane nearest-neighbor coupling [see Fig. 1(a)]. It determines the in-plane $v_{F}$ and in-plane

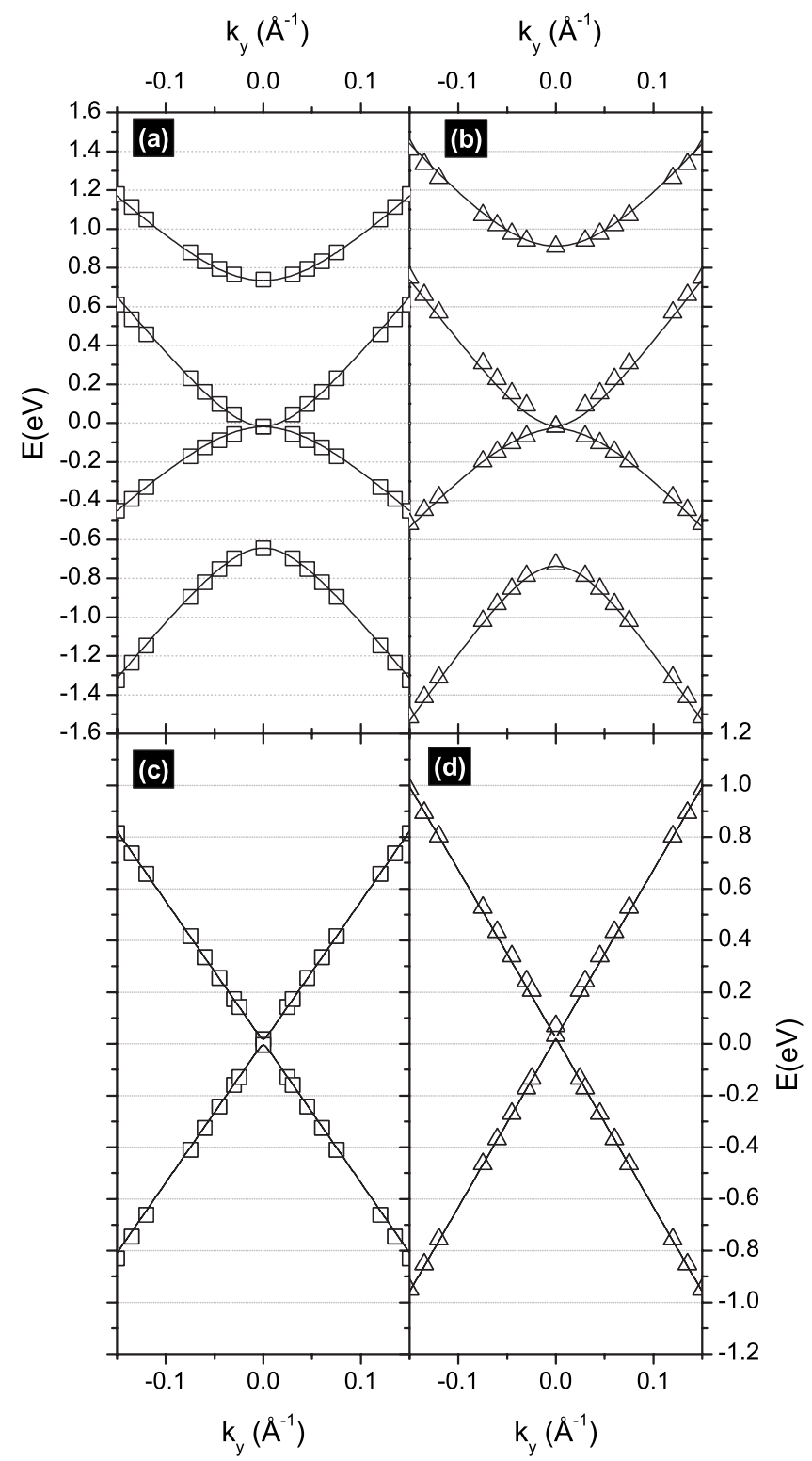

FIG. 3. Upper panel: The TB fits along $k_{y}$ direction at $k_{z}=0(K$ point) for (a) LDA and (b) $G W$. Lower panel: the $k_{y}$ dispersion at $k_{z}=0.47 \AA^{-1}$ ( $H$ point) for (c) LDA and (d) $G W$. $\square$ denotes LDA calculations and $\triangle$ denotes $G W$ calculations that were used for the fitting (taken from Ref. 13).

bandwidth, which is proportional to $\gamma_{0}^{1}-2 \gamma_{0}^{3}$ (Refs. 26 and 40) (or proportional to $\gamma_{0}^{\prime}$ in the SWMC Hamiltonian). In Fig. 4 the trigonal warping effect is illustrated by plotting equienergy contours for $k_{z}=0$ with (a) TB-LDA and (b) TB- $G W$ for energies of $E=0.1 \mathrm{eV}, \ldots, 0.5 \mathrm{eV}$ in steps of $0.1 \mathrm{eV}$. The trigonal warping effect is determined by $\gamma_{3}$ (or proportional to $\gamma_{3}^{\prime}$ in the SWMC Hamiltonian). In order to give a numerical estimate of the trigonal warping, we evaluate the distances between the $K$ point to the equienergy contour with $E=0.4 \mathrm{eV}$ for the TB-LDA and the TB- $G W$ calculations. For the TB-LDA calculation we obtain $0.147 \AA^{-1}$ $\left(0.117 \AA^{-1}\right)$ for the distance along $K M(K \Gamma)$ direction. For TB- $G W$ the corresponding values are $0.135 \AA^{-1}$ $\left(0.100 \AA^{-1}\right)$ along $K M(K \Gamma)$. A good measure of the trigonal 


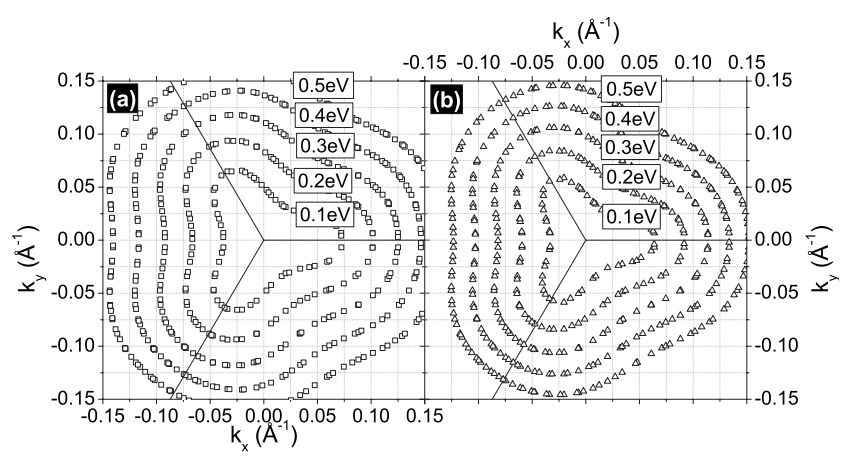

FIG. 4. The TB fits of equienergy contours at $k_{z}=0$ for (a) TBLDA and (b) TB- $G W$.

warping effect is the ratio of $K M$ to $K \Gamma$ distances, which yield 1.256 and 1.347 for TB-LDA and TB- $G W$, respectively. Thus, the trigonal warping effect is about $10 \%$ larger for the TB- $G W$ compared to the TB-LDA (for the $0.4 \mathrm{eV}$ contour).

\section{THREE-DIMENSIONAL QUASIPARTICLE DISPERSION AND DOPING-DEPENDENT FERMI SURFACE OF GRAPHITE}

In Sec. $\mathrm{V}$ we have shown that the $\pi$ bandwidth increases by $20 \%$ when going from TB-LDA to TB- $G W$. This is important especially for the transport measurements (such as STS and contact measurements) and the ARPES results. Optical properties such as the $\pi \rightarrow \pi^{*}$ transition that plays an important role in optical absorption and resonance Raman will also be affected, but in this case the situation is more complicated since the formation of excitons with a finite binding energy will cancel the self-energy corrections of TB- $G W$ to the optical gap. However, for two-photon photoexcitation spectroscopies, the binding energy of an exciton in the $2 p$ state has a smaller value than in the $1 s$ state, and thus, the optical gap should be well described by TB- $G W$.

In Fig. 5 we show the complete in-plane QP band structure for (a) $k_{z}=0$ ( $K$ point) and (b) $k_{z}=0.46 \AA^{-1}$ ( $H$ point)
TABLE III. Energy values of the $G W$ calculation and the $3 \mathrm{NN}$ TB fit (TB- $G W$ ) at high symmetry points in the $3 \mathrm{D}$ BZ of graphite (all values in units eV). The symbols $\pi_{1}-\pi_{4}$ denote the four $\pi$ bands of graphite. The TB- $G W$ have been calculated with the $3 \mathrm{NN}$ TB- $G W$ parameters from Table I.

\begin{tabular}{lccccc}
\hline \hline Point & Method & $\pi_{1}$ & $\pi_{2}$ & $\pi_{3}$ & $\pi_{4}$ \\
\hline$\Gamma$ & $G W$ & -9.458 & -7.257 & 12.176 & 12.541 \\
& TB- $G W$ & -9.457 & -7.258 & 12.184 & 12.540 \\
$M$ & $G W$ & -3.232 & -2.441 & 1.655 & 2.491 \\
& TB- $G W$ & -3.216 & -2.457 & 1.656 & 2.495 \\
$K$ & $G W$ & -0.736 & -0.025 & -0.025 & 0.917 \\
& TB- $G W$ & -0.728 & -0.024 & -0.024 & 0.909 \\
$H$ & $G W$ & 0.020 & 0.020 & 0.025 & 0.025 \\
& TB- $G W$ & 0.020 & 0.020 & 0.025 & 0.025 \\
\hline \hline
\end{tabular}

calculated by the 3NN TB Hamiltonian. In the $\Gamma K M$ plane two valence bands $\left(\pi_{1}\right.$ and $\left.\pi_{2}\right)$ and two conduction bands $\left(\pi_{3}\right.$ and $\left.\pi_{4}\right)$ can be seen, and in the whole $H A L$ plane the two valence (conduction) bands are degenerate. The ab initio $G W$ values are compared to the $3 \mathrm{NN}$ TB- $G W$ calculation in Table III. It can be seen that the fit reproduces the $a b$ initio calculations with an accuracy of $\sim 10 \mathrm{meV}$ in the BZ center and an accuracy of $1 \mathrm{meV}$ along the $K H$ axis, close to $E_{F}$.

Close to $E_{F}$ the $3 \mathrm{NN}$ Hamiltonian is identical to the SWMC Hamiltonian. Thus, for evaluation of the Fermi surface and the doping dependence on $E_{F}$ in the dilute limit, we use the SWMC Hamiltonian. The weakly dispersing band that crosses $E_{F}$ is responsible for the Fermi surface and the electron and hole pockets. This energy band is illustrated in Fig. 6(a) where we show that the TB- $G W$ fit has an accuracy of $1 \mathrm{meV}$. The minority pocket that is a result of the steeply dispersive energy band close to $H$ is shown in Fig. 6(b).

The QP dispersion close to $E_{F}$ is shown in Fig. 7 for [(a), (b)] $K$ and [(c), (d)] $H$ points. The dispersion around the $K$ point is particularly complicated: there are four touching points between valence and conduction bands. Three touching points between the valence and conduction bands exist in

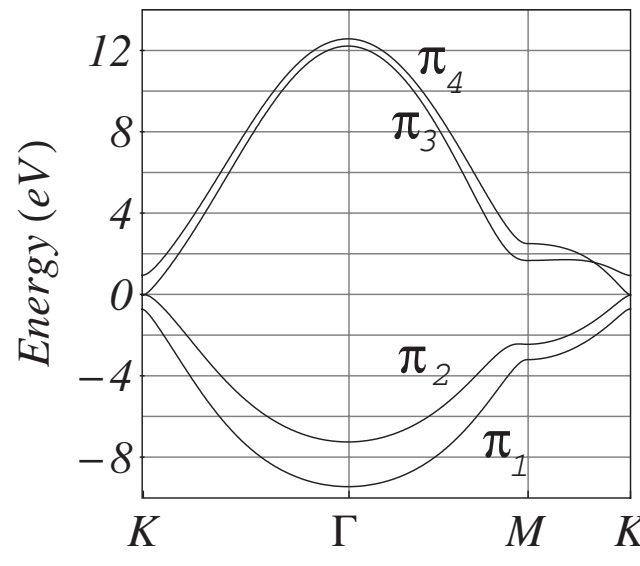

(a)

\section{electron wavevector}

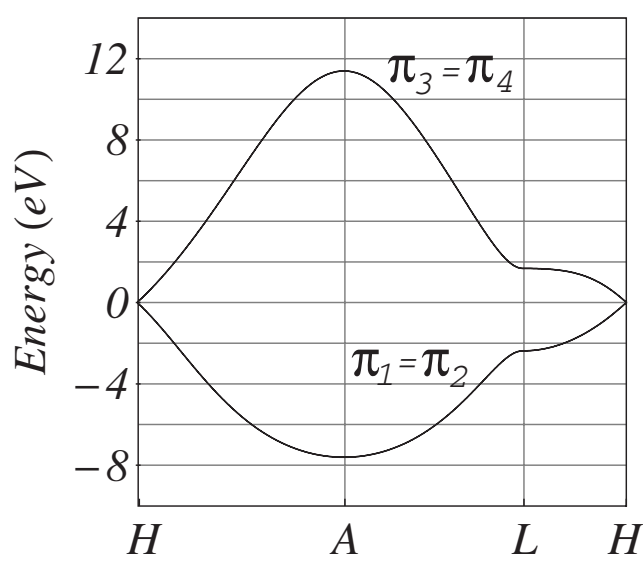

(b)

FIG. 5. The in-plane quasiparticle dispersion for (a) $k_{z}=0$ and (b) $k_{z}=0.47 \AA^{-1}$ calculated by TB-GW. The symbols $\pi_{1}-\pi_{4}$ denote the four $\pi$ bands of graphite. 

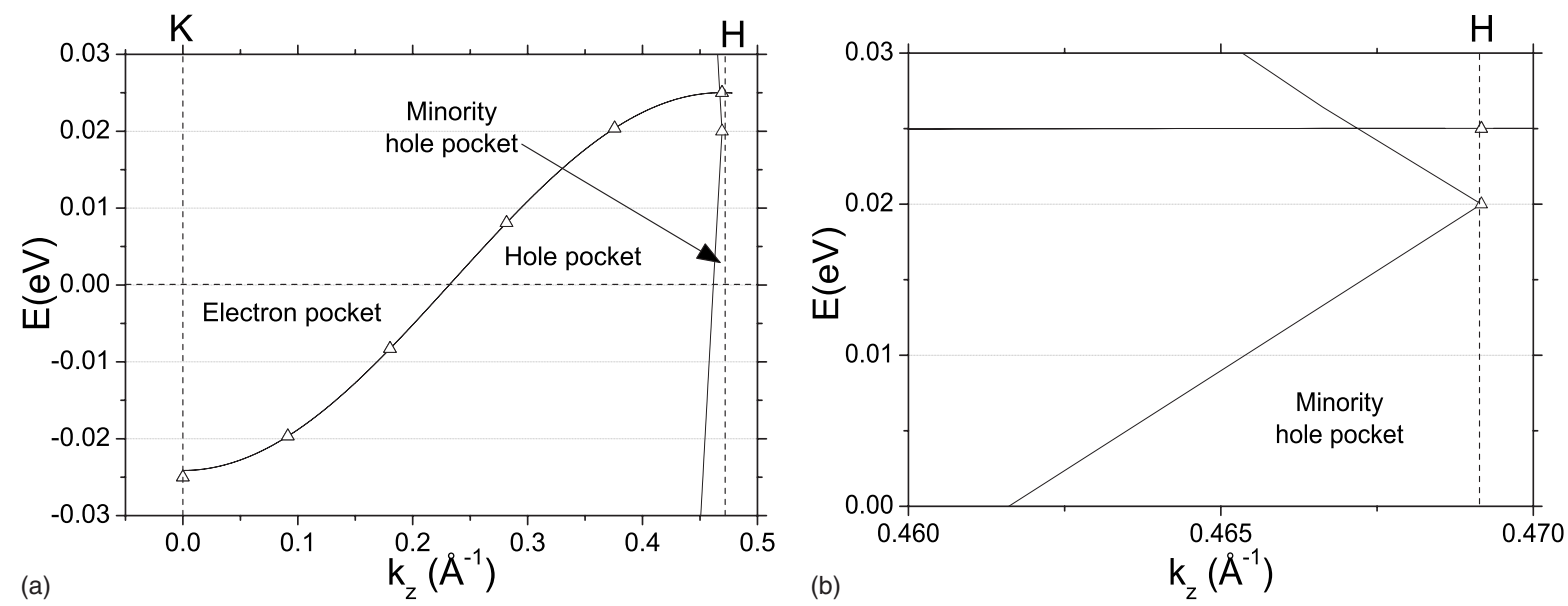

FIG. 6. (a) The weakly dispersive band that is responsible for the formation of electron and hole pockets. $\triangle$ denotes $G W$ calculations (with the impurity doping level adjusted to reproduce the experimental gap at $H$ point) and the line the TB- $G W$ fits. (b) shows a magnification of the minority hole pocket that is caused by the steeply dispersive band close to $H$.

the close vicinity to $K$ at angles of $0^{\circ}, 120^{\circ}$, and $240^{\circ}$ away from $k_{x}$ (i.e., the $K M$ direction). The fourth touching point is exactly at $K$ point. The touching points arise from the semimetallic character of graphite: there are two parabolas (VB and $\mathrm{CB}$ ) that overlap by about $20 \mathrm{meV}$. For example the

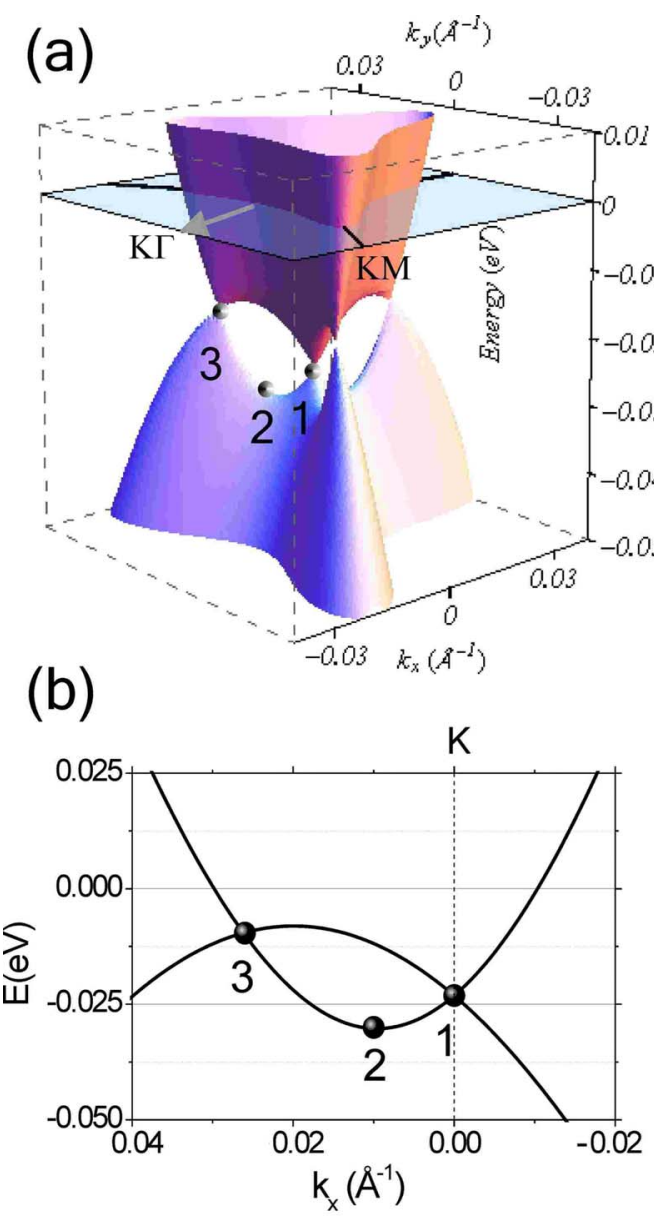

bottom of the CB is denoted by point 2 in Figs. 7(a) and 7(b). At $H$ point shown in Figs. $7(\mathrm{c})$ and $7(\mathrm{~d})$ the energy-band structure becomes simpler: The $\pi$ and $\pi^{*}$ bands are each doubly generate, and their dispersion is rather isotropic around $H$ (the trigonal warping effect in the $k_{x} k_{y}$ plane is a

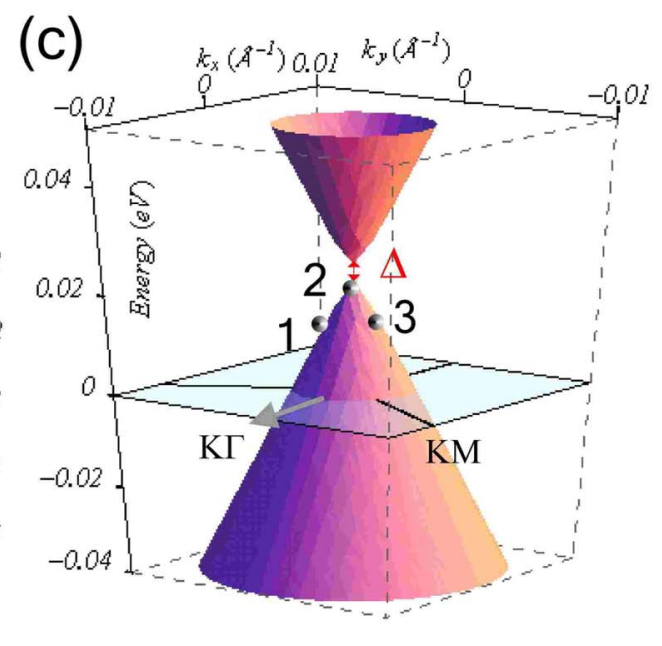

(d)

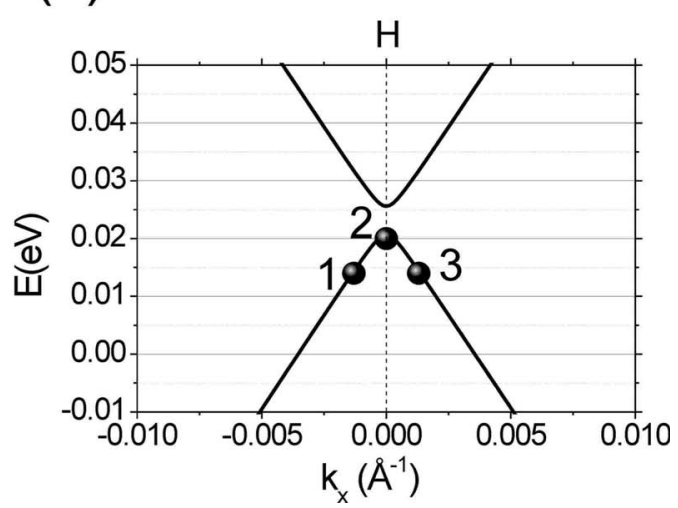

FIG. 7. (Color online) TB- $G W$ QP band structure around [(a), (b)] $K$ point and [(c), (d)] $H$ point. The points 1,2 , and 3 denote the dispersion we use to determine electron and hole masses (see Fig. 9). The points 1, 2, and 3 span a parabola. In [(a), (b)] the parabola is along the $K M$ direction with heaviest electron masses and in [(c), (d)] the hole masses are isotropic around $H$. 

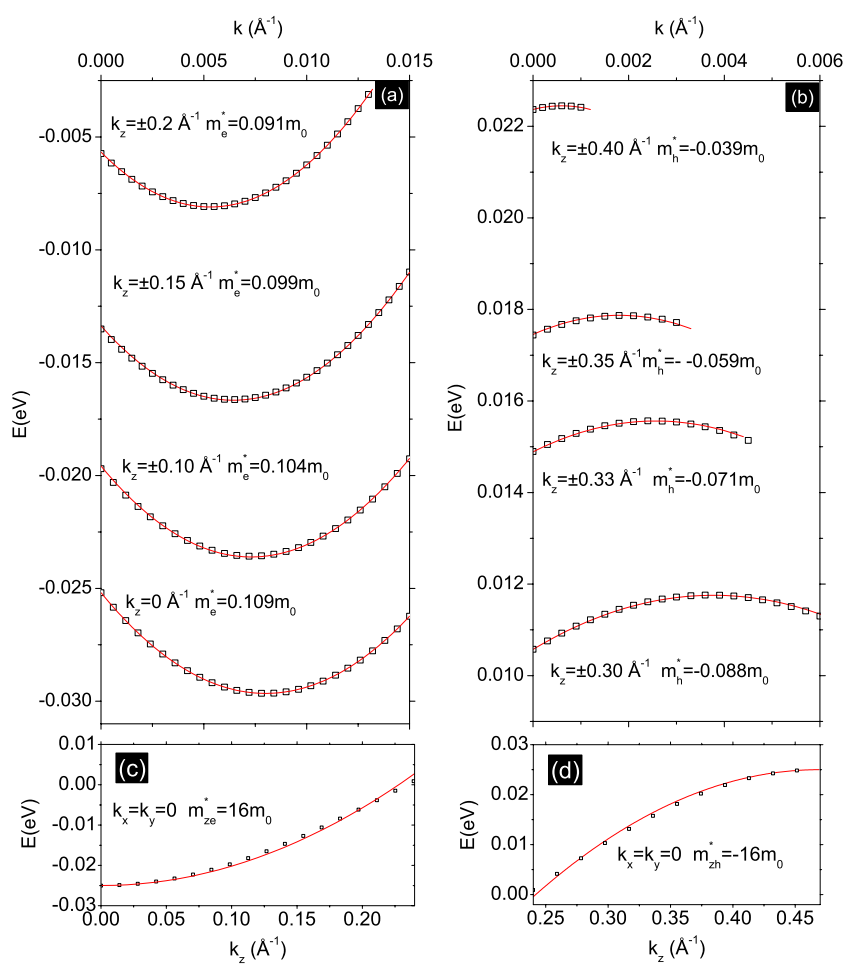

FIG. 8. (Color online) Evaluation of the in-plane (a) electron and (b) hole masses around $K$ and $H$, respectively. The dispersions along the parabolas depicted by points 1-2-3 in Fig. 7 are taken for evaluation of the masses. The effective masses perpendicular to the layers in $z$ direction are shown in (c) for electrons and in (d) for holes. $\square$ correspond to TB- $G W$ values and the lines are parabolic fits.

minimum in the $A H L$ plane and a maximum in the $\Gamma K M$ plane). It is clear that the energy bands do not touch each other and the dispersion is not linear but parabolic with a very large curvature (and, hence, a very small absolute value of the effective mass; see Fig. 8) at $H . E_{F}$ lies $20 \mathrm{meV}$ below the top of the VB and the energy gap $\Delta$ is equal to $5 \mathrm{meV}$. It is interesting to note that a larger value of $\Delta$ would bring the top of the VB below $E_{F}$. The horizontal cuts through the dispersions in Fig. 7 at $E=E_{F}$ (blue area) give cross-sectional areas of an electronlike Fermi surface at $k_{z}=0(K)$ and a holelike Fermi surface at $k_{z}=0.47 \AA^{-1}$ ( $H$ point), consistent with a semimetallic behavior.

The effective in-plane massses for electrons $\left(m_{e}^{*}\right)$ and holes $\left(m_{h}^{*}\right)$ are evaluated along the parabola indicated in Fig. 7 by points 1,2 , and 3 . This parabola lies in the plane spanned by $K M$ and $H L$ and can, thus, be considered an upper limit for the effective mass since the dispersion is flat in this direction, as can be seen in Fig. 7(a). For $m_{e}^{*}$ the center of the parabola is chosen to be the bottom of the $\mathrm{CB}$, i.e., point 2 in Fig. 7(a). Similarly for $m_{h}^{*}$ the center of the parabola is chosen to be the top of the VB, i.e., point 2 in Fig. 7(b). Due to the larger curvature of the hole bands, the absolute value of $m_{e}^{*}$ is larger than $m_{h}^{*}$. The $k_{z}$ dependence of $m_{e}^{*}$ and $m_{h}^{*}$ is shown in Figs. 8(a) and 8(b), respectively. For the effective mass in the $z$ direction we fit a parabola for the weakly dispersing band in direction perpendicular to the layers and get $m_{z e}^{*}=16 m_{0}$ and $m_{z h}^{*}=-16 m_{0}$ for the effective elec-

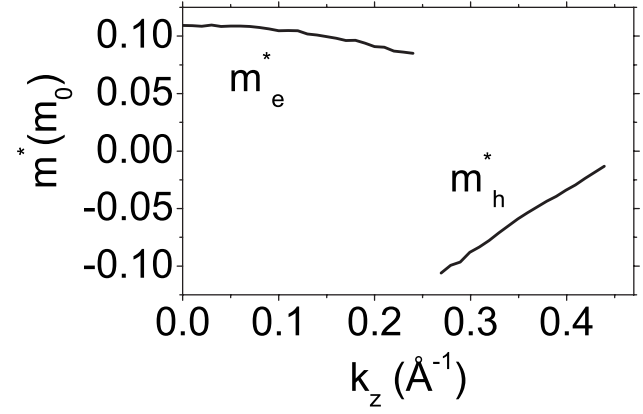

FIG. 9. The $k_{z}$ dependence of the in-plane electron mass $\left(m_{e}^{*}\right)$ and hole mass $\left(m_{h}^{*}\right)$ calculated by TB- $G W$. The masses are evaluated along the parabolas as shown in Fig. 8.

tron and hole masses perpendicular to the layers, respectively. Figures 8(c) and 8(d) shows the TB- $G W$ along $k_{z}$ for the electron and hole pocket, respectively. The parabolic fits that were used to determine the effective masses are shown along with the calculation.

The $k_{z}$ dependence of $m_{e}^{*}$ and $m_{h}^{*}$ are shown in Fig. 9. For this purpose we evaluated the heavy electron mass of the parabolic subbands, as shown in Figs. 7 and 8. It is clear that $m_{e}^{*}$ has a weak $k_{z}$ dependence and $m_{h}^{*}$ strongly depends on the value of $k_{z}$. This is obvious since exactly at $H$ point, the value of $m_{h}^{*}$ has a minimum. For a finite value of the gap $\Delta$, the value of $m_{h}^{*}$ also remains finite.

We now discuss the whole 3D Fermi surface. The volume inside the surface determines the low-energy free carrier plasmon frequencies and the electrical conductivity. The trigonal warping has little effect on the volume inside the electron and hole pocket. When we set $\gamma_{3}=0$, then the Fermi surface is isotropic around $K H$ axis. The simplification of $\gamma_{3}=0$ results in little change in the volume. For $\gamma_{3} \neq 0$, there are touching points of the electronlike and holelike Fermi surfaces. ${ }^{28}$ The touching points (or legs) are important for understanding the period for de Haas-van Alphen effect and the large diamagnetism in graphite. ${ }^{39}$ However, for the calculation of the number of carriers, they are not crucial, and thus the Fermi surface calculated with $\gamma_{3}=0$ can be used for the evaluation of the electron density, $n_{e}$, and the hole density, $n_{h}$. In this case, the cross section of the Fermi surface $A\left(k_{z}\right)$ has an analytical form. The number of electrons per $\mathrm{cm}^{3}$ is given by $n_{e}=4 \times 10^{24} \times f_{u} / v_{u c}$ with $f_{u}=v_{e} / v_{b z}$, where $v_{e}$ is the electron pocket volume and $v_{b z}$ the BZ volume. $v_{u c}$ is the unit-cell volume in $\AA^{-3}$. Similarly, by replacing $v_{e}$ with $v_{h}$ (the volume of the whole pocket) one can obtain $n_{h}$, the number of holes per $\mathrm{cm}^{3}$. The critical quantities are $v_{e}$ and $v_{h}$, and they are obtained by integrating the cross section of the Fermi surface $A\left(k_{z}\right)$ along $k_{z}$. The analytical expression and their dependence on the TB parameters is given in Ref. 30. This yields plasmon frequencies of $\hbar \omega_{a}$ $=\hbar \sqrt{n_{e} e^{2} /\left(m_{e}^{*} \epsilon_{0} \epsilon_{a}\right)}=113 \mathrm{meV}$ for plasmon oscillation parallel to the graphene layers and $\hbar \omega_{c}=\hbar \sqrt{n_{e} e^{2} /\left(m_{z e}^{*} \epsilon_{0} \epsilon_{c}\right)}$ $=19 \mathrm{meV}$ for plasmon oscillation perpendicular to the layers. Here $\epsilon_{a}=5.4$ (Ref. 41) and $\epsilon_{c}=1.25$ (Ref. 42) are adopted for the dielectric constants parallel and perpendicular to the graphene layers, respectively. We have made two simplifications: first we do not consider a finite value of temperature 

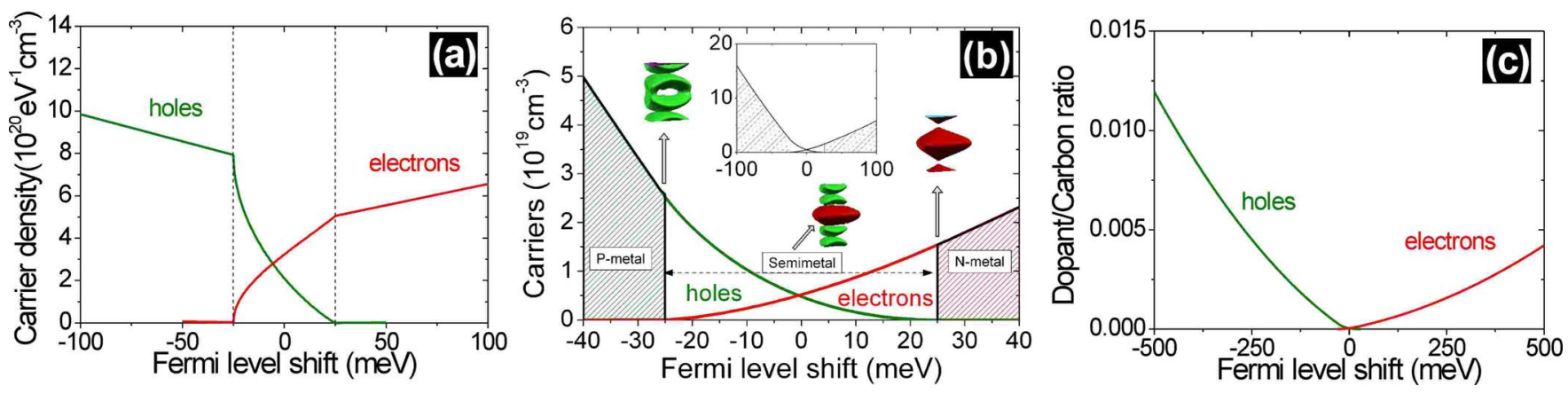

FIG. 10. (Color online) Doping dependence of (a) the electron and hole carrier density, (b) the number of electrons and holes, and (c) the stochiometric dopant to carbon ratio. The red (green) lines represent electron (hole) carriers.

( $\omega_{a}$ and $\omega_{c}$ are the plasmon frequencies at $0 \mathrm{~K}$ ), and second we used an effective mass averaged over the whole $k_{z}$ range of the pockets as shown in Figs. 8 and 9.

Next we discuss the doping dependence of the electronic properties in the so-called dilute limit, which refers to a very low ratio of dopant/carbon atoms. Here we use a method described previously ${ }^{30}$ employing the TB- $G W$ parameters from Table II. This allows us to calculate the doping dependence of $n_{e}$ and $n_{h}$. The analytical formula for the $E_{F}$-dependent cross section of the Fermi surface, $A\left(k_{z}\right)$ (given in Ref. 30), is integrated for different values of $E_{F}$. By integrating $d A\left(k_{z}\right) / d E_{F}$ along $k_{z}$ we obtain the carrier density per electronvolt. The ratio of dopant to carbon is given by $r=n \times v_{u c} / 4 f$, where $n=n_{e}$ for electron doping and $n=n_{h}$ for hole doping. Here $f$ is the charge-transfer value per dopant atom to carbon. Although there are some discussions about the value of $f$, it is was recently found for potassium-doped graphite that $f \sim 1.43,44$ In Fig. 10 (a) we show the doping dependence of the carrier densities. It is clear that at $E_{F}$ $= \pm 25 \mathrm{meV}$, we have a discontinuity in the carrier density, and this is associated to the $E_{F}$ at which the electron or hole pocket is completely filled. Since the density of states decreases suddenly after the pockets are filled, the kink in the density of states appears. This also marks the transition from a two-carrier regime to a single-carrier regime. In Fig. 10(b) we show $n_{e}$ and $n_{h}$. It is clear that at $E_{F}=0$ the number of holes equals the number of electrons. At $E_{F}=25 \mathrm{meV}$, we have no more holes and thus a transition from a semimetal to a metal occurs. In such a metal, the carriers are electrons, hence, an $N$-type metal. Similarly, at $E_{F}=-25 \mathrm{meV}$ we have no more electrons and a semimetal to metal transition occurs in the other direction to $E_{F}$. For this metal, the carriers are holes, hence, a $P$-type metal. These semimetal to metal transitions are important for ambipolar transport in graphite and graphene: they determine the region for the gate voltage in which ambipolar transport is possible. Finally in Fig. 10(c) we plot the ratio of dopant to carbon atoms as a function of $E_{F}$.

It is certainly interesting to monitor the doping induced changes in $n_{e}$ and $n_{h}$ also in the shape of the Fermi surface. In Fig. 11 we show the Fermi surfaces for electron doping and hole doping for $E_{F}=0$ to $\pm 35 \mathrm{meV}$ in steps of $5 \mathrm{meV}$. It is clear that at $\pm 25 \mathrm{meV}$ a single carrier regime dominates, as indicated by the two different colors (red for electrons and green for holes). This is consistent with Fig. 10(b) where the integrated electron (hole) densities disappear at $E_{F}$ $=25 \mathrm{meV}\left(E_{F}=-25 \mathrm{meV}\right)$.

\section{FEW LAYER GRAPHENE}

The 3 NN TB- $G W$ set of parameters fits the whole $k_{z}$ range of the 3D graphite BZ. Thus the set can be transferred for the calculation of QP dispersions of stacked $s p^{2}$ FLGs with $N$ layers $(N=1,2, \ldots)$. The transferability of TB parameters is a result of the fact that the lattice parameters of FLGs and graphite are almost identical. ${ }^{45}$ We can use the matrix elements shown in Fig. 1(a) also for FLGs; for $N=1$ only $\gamma_{0}^{1}, \gamma_{0}^{2}$, $\gamma_{0}^{3}, s_{0}^{1}, s_{0}^{2}, s_{0}^{3}$, and $E_{0}$ are needed and this results in the graphene monolayer case. For $N=2$ the parameters $\gamma_{2}$ and $\gamma_{5}$ are not needed since they describe next-nearest-neighbor interactions which do not exist in a bilayer. We use the set of TB parameters given in Table I and the Hamiltonians given in Sec. II and Appendix. In Fig. 12 we show the bilayer $(N$ $=2)$, the trilayer $(N=3)$, and the quadlayer $(N=4)$ calculated with TB- $G W$. Figures 12 (a) and 12(b) show the electron dispersion of the bilayer. The separation between $\mathrm{CB}$ (VB) to the Fermi level is proportional to $\gamma_{1}$ (Ref. 46), and thus it is clear that the TB- $G W$ also gives a separation between the VB of about $20 \%$ larger than that of LDA. Furthermore the slope of all bands becomes steeper for TB- $G W$ with respect to

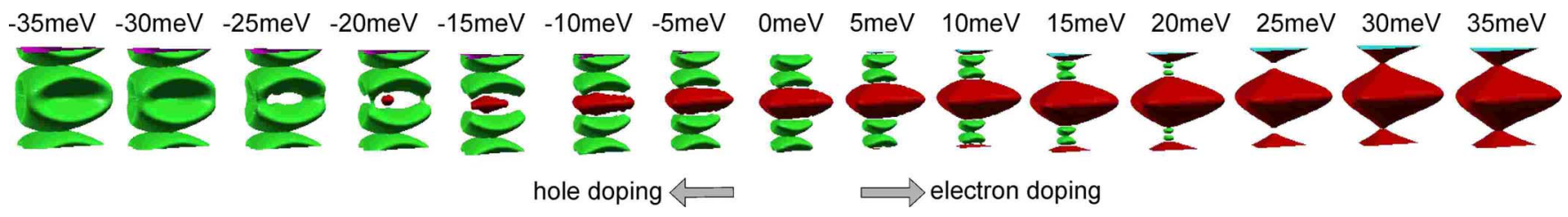

FIG. 11. (Color online) The Fermi surface of doped graphite in the dilute limit for electron doping and hole doping. The red (green) surfaces are the electron (hole) pockets of the Fermi surface. It is clear that at $\pm 25 \mathrm{meV}$ we have a semimetal to metal transition. 

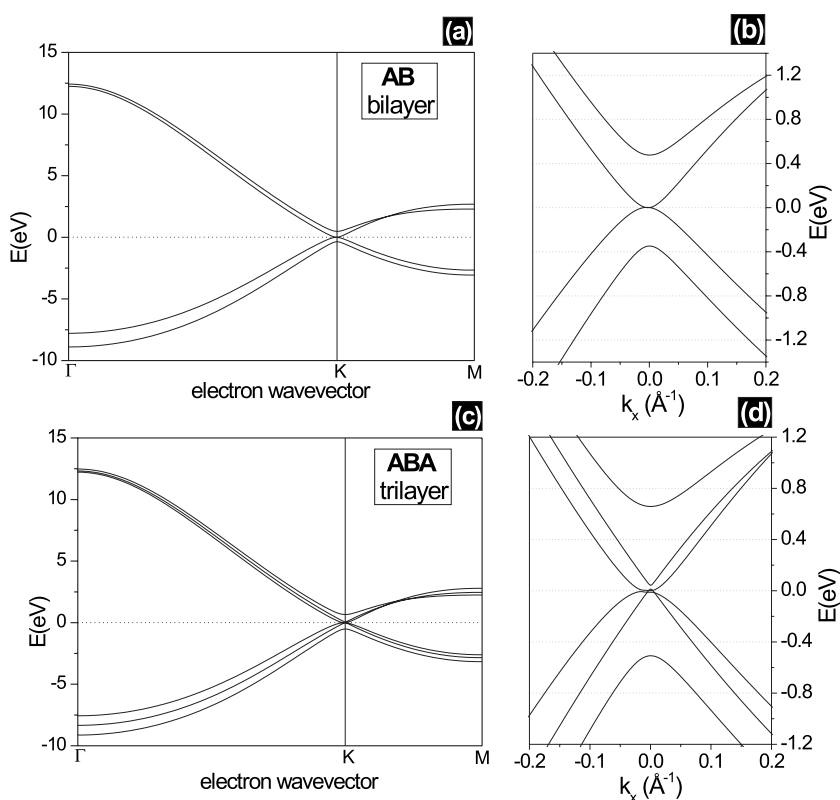

(c)
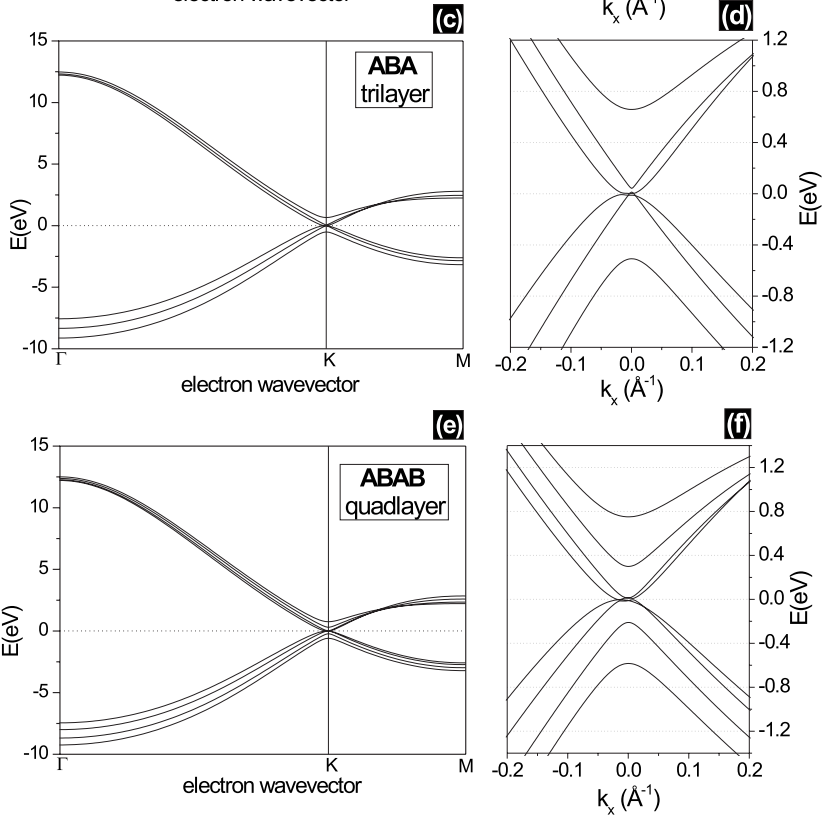

FIG. 12. Quasiparticle $\pi$ bands for [(a), (b)] bilayer, [(c), (d)] trilayer, and [(e), (f)] quadlayer graphene. The right panels show a magnification of the band structure around $K$ point. These band structures have been calculated by $3 \mathrm{NN}$ TB- $G W$ using the parameters from Table I. The corresponding Hamiltonian and overlap matrices are given in Appendix.

TB-LDA calculations. This is also responsible for the increase in $v_{F}$ of FLGs (similar to the graphite case, when going from LDA to $G W$ ). The same argument is the case for the trilayer and quadlayer. It is interesting that the QP dispersion measured by ARPES (Ref. 9) is in better agreement with the TB- $G W$ rather than the LDA calculations performed.

The low-energy dispersion relation of FLGs are particularly important for describing transport properties. From the calculations, we find that all FLG has a finite density of states at $E_{F}$. The trilayer has a small overlap at $K$ point between valence and conduction band (i.e., semimetallic). This property might be useful for devices with ambipolar transport properties. Our results are in qualitative agreement with the LDA calculations. ${ }^{47}$

It can be seen that a linear (Dirac-type) band appears for the trilayer (and also for all other odd-numbered multilayers). This observation is in agreement to previous calculations and is relevant to an increase in the orbital contribution to diamagnetism. ${ }^{48}$

Since TB allows for rapid calculation of the QP bands, the transition from FLG to bulk graphite can be analyzed. Even

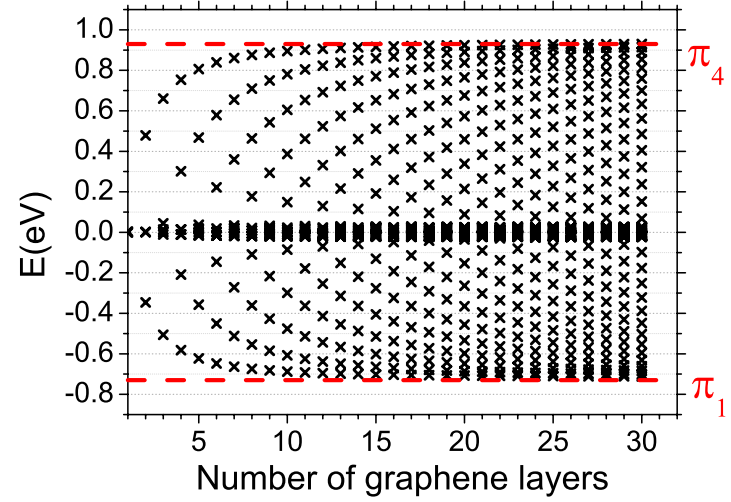

FIG. 13. (Color online) Evolution of the eigenvalue spectrum for FLG from $N=1 \ldots 30$ graphene layers calculated by TB- $G W$ at the $K$ point. The dashed lines labeled by $\pi_{1}$ and $\pi_{4}$ denote the lower and upper limits of the total bandwidth at $K$ point in 3D graphite. A pattern that connects the first, second, etc. energies of FLG is emerging (see text).

in the case of $N=30$, the solution of Eq. (4) for the $60 \times 60$ Hamiltonian and overlap matrices takes only $\sim 10 \mathrm{sec}$ on a Pentium workstation per $k$ point. In Fig. 13 we show the eigenvalue spectrum for FLG with $N=1, \ldots, 30$. As we increase $N$ and, hence, the number of $\pi$ bands, the bandwidth also increases and approaches that of bulk graphite. It can be seen that for $N>15$, the total bandwidth is that of bulk graphite. Interestingly, the energies of the $\pi$ bands group together and form families of the highest, second-highest, etc. energy eigenvalue at $K$. With increasing number of layers, a given family approaches the limit for bulk graphite. Such a family pattern is a direct consequence of the $A B$ stacking sequence in FLG, and it might be accessible to optical spectroscopy similar to the fine structure around $K$ point that has been observed in bulk graphite. ${ }^{49}$

In the limit of one isolated graphene layer, the TB- $G W$ fit no longer exactly reproduces the slope of the $\pi$ bands. The reason is that screening in an isolated (2D) graphene sheet is considerably weaker than in 3D bulk graphite. Therefore, the electron-electron interaction is stronger in single-layer graphene and leads to a stronger renormalization of the bands. For example, in graphite, the gap at $H$ is renormalized by $13 \%$, while in single-layer graphene, the corresponding gap at $M$ is renormalized by $21 \% .^{50}$ Note, however, that graphene is usually deposited on a substrate. ${ }^{1,3,5}$ Thus, the dielectric screening of the substrate will contribute to the renormalization and cancel to some extent the very strong $G W$ renormalization of the imaginary isolated graphene sheet.

\section{DISCUSSION}

We first discuss the QP band structure and relation to recent ARPES experiments. From several experimental works it is clear that the LDA bands need to be scaled in order to fit the experiments. ${ }^{12-14}$ Our set of TB parameters quantitatively describes the QP dispersions of graphite and FLG. The scaling is mainly reflected in an increase in $\gamma_{0}^{1}, \gamma_{0}^{2}$, $\gamma_{0}^{3}$, and $\gamma_{1}$, the in-plane and out-of-plane coupling, respec- 
tively. It, thus, can be used to analyze ARPES of both pristine and doped (dilute limit) graphite and FLG. Most importantly, the correlation effects increase $v_{F}$, the Fermi velocity when going from LDA to $G W$. For the TB- $G W$ calculation, $v_{F}=1.01 \times 10^{6} \mathrm{~ms}^{-1}$, which is in perfect agreement to the values from ARPES that is equal to $v_{F}=1.06 \times 10^{6} \mathrm{~ms}^{-1} .^{13}$ The question why LDA works for some metals but fails to give the correct $v_{F}$ and energy-band dispersion in semimetallic graphite arises. In graphite, the contribution of the electron-electron interaction to the self-energy is unusually large. The reason for this is the small number of free carriers to screen efficiently the Coulomb interaction. In most other metals, the density of states at $E_{F}$ has a much larger $(\sim 1000$ times $)$ value than in graphite and the screening lengths are shorter. Hence the LDA is a good description for such a material but it fails in the case of graphite. Another parameter that illustrates the quality of the TB- $G W$ to reproduce the experimental QP dispersion is $\delta$, the band splitting at $K$ point. For TB- $G W$, we obtain $\delta=0.704 \mathrm{eV}$ and the ARPES gives $\delta=0.71 \mathrm{eV}^{13}$

The TB parameters of the SWMC Hamiltonian have been fitted in order to reproduce double-resonance Raman spectra. ${ }^{21}$ As a result they obtained that $\gamma_{3}^{\prime}$ (see Fig. 1) has a value of $0.1 \mathrm{eV}$, while that for graphite that we obtain in this paper is $0.274 \mathrm{eV}$ (see Table II) and the value that was fit to transport experiments is $0.315 \mathrm{eV},{ }^{28,51}$ which is also in perfect agreement to ARPES experiments of graphite single crystals. ${ }^{51}$ We now discuss a possible reason for this discrepancy. The fitting procedure in Ref 21 depends on the choice of the phonon-dispersion relation of graphite. It is important to note that recently a Kohn anomaly has been directly observed by inelastic x-ray scattering experiments using synchrotron radiation, ${ }^{52}$ which has a steeper slope of the TO phonon branch at $K$ point in contrast to previous measurements. ${ }^{53}$ Thus the assumption of the correct phonondispersion relation is crucial for obtaining the correct bandstructure parameters.

Next we discuss the present QP electronic energy-band structure in relation to optical spectroscopies such as optical absorption spectroscopy (OAS) and resonance Raman spectroscopy. The optical spectroscopies probe the joint density of states (JDOS) weighted with the dipole matrix elements. Peaks in the OAS are redshifted when compared to the JDOS of the QP dispersion if excitons are created. By comparing the QP dispersion with OAS experiments, ${ }^{49,54,55}$ we now estimate a value for exciton binding energies in graphite. In general many resonant states with $k$ contribute to OAS, but a fine structure in OAS of bulk graphite measured in reflection geometry was observed by Misu et al. ${ }^{49}$ which was assigned to two specific transitions around $K$ : the $A_{1}$ transition between the lower VB and states just above $E_{F}$ and the $A_{2}$ transition between the upper VB and the upper $\mathrm{CB}$. The experimental energies they found were $E_{A 1}^{\exp }=0.669 \mathrm{eV}$ and $E_{A 2}^{\exp }=0.847 \mathrm{eV}$. The TB- $G W$ dispersion yields energies $E_{A 1}^{G W}=0.722 \mathrm{eV}$ and $E_{A 2}^{G W}=0.926 \mathrm{eV}$ [see Fig. 2(b)]. Assuming one exciton is created for the $A 1(A 2)$ transition, this yields exciton binding energies of $E_{A 1}^{G W}-E_{A 1}^{\exp } \sim 50 \mathrm{meV}$ and $E_{A 2}^{G W}-E_{A 2}^{\exp } \sim 80 \mathrm{meV}$ for a $K$-point exciton in bulk graphite. Such a value for the exciton binding energies most probably increases when going from bulk graphite to FLGs due to confinement of the exciton wave function in $z$ direction.

Concerning the value of $\Delta$, the gap at $H$, several experimental values exist and magnetoreflectance experiments suggest $\Delta=5 \mathrm{meV}$. This is in disagreement to the calculated values obtained for pristine graphite. ${ }^{13}$ However, when the doping level is slightly increased, $\Delta$ becomes smaller, and we thus, fixed a doping level in the $a b$ initio calculation that reproduces the experimental $\Delta .{ }^{34}$ While some of the variations may be explained by the sample crystallinity in $z$ direction, it is also conceivable that small impurities are responsible for the discrepancy.

Next we discuss a possibility to measure the free carrier plasmon frequencies of pristine and alkali-metal-doped graphite by high-resolution energy electron-loss spectroscopy (HREELS) or inelastic tunneling spectroscopy. The electron concentration inside the pockets very sensitively affects the plasmon frequencies. In principle the charge carrier plasmons should also appear as a dip in optical reflectivity measurements, but due to the small relative change in intensity they have not been observed so far. Due to the small size of the pockets, the number of charge carriers and, hence, the conductivity and plasmon frequencies are extremely sensitive to temperature and doping. Experimentally observed plasmon frequencies for oscillations parallel to the graphene layers are $\hbar \omega_{a}=128 \mathrm{meV}$ (Ref. 56) and for oscillations perpendicular to the layers are $\hbar \omega_{c}=45-50 \mathrm{meV} .{ }^{42,56-58}$ These values for $\hbar \omega_{a}$ and $\hbar \omega_{c}$ agree reasonably well with our derived value considering that we make the crude estimation of $\hbar \omega_{a}$ and $\hbar \omega_{c}$ at $0 \mathrm{~K}$ while experiments were carried out at room temperature. We also used average effective masses of the electron pocket in order to determine the plasmon frequency, while in fact there is a $k_{z}$ dependence (see Fig. 9). We also note that the experimental literature values for $\epsilon_{a}$ and $\epsilon_{c}$ have a rather wide range, e.g., $\epsilon_{c}=3.4$ (Ref. 59) from reflectivity measurements and $\epsilon_{c}=5.4$ (Ref. 41) from energy electron-loss spectroscopy (EELS).

The temperature dependence of $\omega_{c}$ was measured by Jensen et al. $^{42}$ by HREELS, and they observed a strong $T$ dependence for $\omega_{c}$, which was attributed to changes in the occupation and, thus, number of free carriers with $T$. The observed plasmon energy was rising from 40 to $100 \mathrm{meV}$ in a temperature range of 100 to $400 \mathrm{~K}$. A similar effect might be observed by HREELS of doped graphite as a function of doping level. It would be interesting to study the evolution of the plasmon frequency with doping level. With our current understanding of the low-energy-band structure we predict a semimetal to metal transition at a Fermi level shift of $E_{F}$ $\sim 25 \mathrm{meV}$. At this doping level, the hole pocket is completely filled with electrons and disappears and the electron pocket has roughly doubled in size, and $\omega_{a}$ and $\omega_{c}$ should increase by a factor of $\sim \sqrt{2}$. Such a transition should be observable by HREELS and might be accompied by interesting changes in the band structure (electron-plasmon coupling), which can also be measured simultaneously by ARPES. Many dc transport properties can be understood with a Drude model for the conductivity, which is inversely proportional to the effective carrier mass. From the ratio of $m_{z e}^{*} / m_{e}^{*}$ and $m_{z h}^{*} / m_{h}^{*}$, we hence expect that the dc electron (hole) conductivity in $z$ direction (out-of-plane) is $\sim 200$ $(\sim 500)$ times less than the in-plane conductivity. The experi- 
TABLE IV. Properties of the electronic band structure of graphite calculated from TB- $G W$ and compared to experiment.

\begin{tabular}{|c|c|c|c|}
\hline Parameter & Symbol & TB- $G W$ & Experimental value(s) \\
\hline Fermi velocity at $H\left(10^{6} \mathrm{~ms}^{-1}\right)$ & $v_{F}$ & 1.01 & $0.91,{ }^{\mathrm{a}} 1.06,{ }^{\mathrm{b}} 1.07,{ }^{\mathrm{c}} 1.02^{\mathrm{d}}$ \\
\hline Splitting of $\pi$ bands at $K(\mathrm{eV})$ & $\delta$ & 0.704 & $0.71^{\mathrm{b}}$ \\
\hline Bottom of $\pi$ band at $A$ point $(\mathrm{eV})$ & $E(A)$ & 7.6 & $8,{ }^{e} 8^{f}$ \\
\hline in-plane electron mass $\left(m_{0}\right)$ & $m_{e}^{*}$ & $0.1\left(k_{z}\right.$ averaged $)$ & $0.084,{ }^{\mathrm{g}} 0.42,{ }^{\mathrm{a}} 0.028,{ }^{\mathrm{c}} 0.1^{\mathrm{b}}$ \\
\hline in-plane hole mass $\left(m_{0}\right)^{\mathrm{h}}$ & $m_{h}^{*}$ & $0.06\left(k_{z}\right.$ averaged $)$ & $0.069,{ }^{\mathrm{a}} 0.03,{ }^{\mathrm{i} j} 0.028,{ }^{\mathrm{c}} 0.04^{\mathrm{b}}$ \\
\hline out-of-plane electron mass $\left(m_{0}\right)$ & $m_{z e}^{*}$ & 16 & \\
\hline out-of-plane hole mass $\left(m_{0}\right)$ & $m_{z h}^{*}$ & -16 & \\
\hline number of electrons at $E_{F}=0\left(10^{18} \mathrm{~cm}^{-3}\right)$ & $n_{e}$ & 5.0 & $8.0,{ }^{\mathrm{a}} 3.1^{\mathrm{k}}$ \\
\hline number of holes at $E_{F}=0\left(10^{18} \mathrm{~cm}^{-3}\right)$ & $n_{h}$ & 5.0 & $3.1,{ }^{\mathrm{a}} 2.7,{ }^{\mathrm{k}} 9.2^{\mathrm{e}}$ \\
\hline Gap at $H$ point $(\mathrm{meV})$ & $\Delta$ & 5 & $5-8^{1, d}$ \\
\hline in-plane plasmon frequency (meV) & $\hbar \omega_{a}$ & 113 & $128,{ }^{\mathrm{m}} 125^{\mathrm{n}}$ \\
\hline out-of-plane plasmon frequency (meV) & $\hbar \omega_{c}$ & 19 & $36,{ }^{\mathrm{o}, \mathrm{p}} 40-50^{\mathrm{o}, \mathrm{m}, \mathrm{n}, \mathrm{q}}$ \\
\hline$A_{1}$ optical transition energy $(\mathrm{eV})$ & $E_{A 1}$ & 0.722 & $0.669^{\mathrm{r}, \mathrm{s}}$ \\
\hline$A_{2}$ optical transition energy $(\mathrm{eV})$ & $E_{A 2}$ & 0.926 & $0.847^{\mathrm{r}, \mathrm{s}}$ \\
\hline $\begin{array}{l}{ }^{\mathrm{a}} \text { Reference } 32 . \\
\text { bReference } 13 . \\
{ }^{\mathrm{c}} \text { Reference } 61 . \\
{ }^{\mathrm{d}} \text { Reference } 62 . \\
{ }^{2} \text { Reference } 63 . \\
{ }^{\mathrm{f}} \text { Reference } 64 . \\
\text { geference } 65 \text {. } \\
{ }^{\mathrm{h}} \text { To compare different notations, we denote } \\
\text { absolute value of } m_{h}^{*} \text {. } \\
{ }^{\mathrm{i}} \text { Reference } 66 \text {. } \\
\text { jMass at the } H \text { point was measured. Note th: } \\
\text { in excellent agreement to our calculated } k_{z} \\
\text { dence of } m_{h}^{*} \text { (see Fig. 9). }\end{array}$ & $\begin{array}{l}\text { the } \\
\text { is is } \\
\text { pen- }\end{array}$ & \multicolumn{2}{|c|}{ 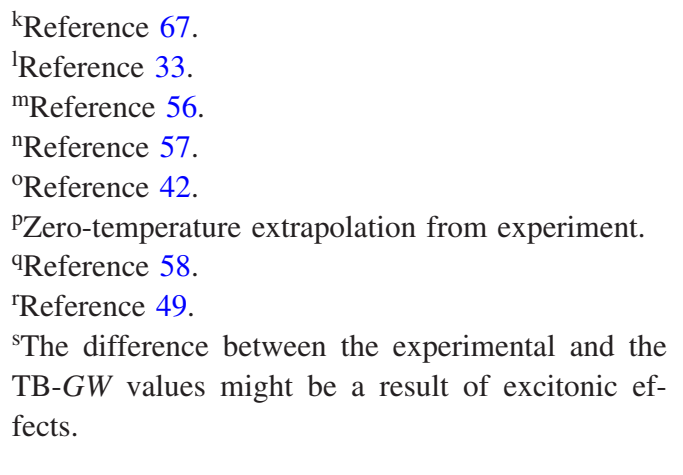 } \\
\hline
\end{tabular}

mental value for the ratio between in-plane and out-of-plane conductivity is $3 \times 10^{3}$ (Ref. 28) and considering the large variation in experimental values even for the in-plane conductivity, ${ }^{28,60}$ it is in reasonable agreement. In Table IV we summarize the electronic band-structure properties such as $v_{F}, \delta$, and the effective masses and compare them to experimental values.

\section{CONCLUSIONS}

In conclusion, we have fitted two sets of TB parameters to first-principles calculations for the LDA and QP $(G W)$ electron energy dispersions of graphite. We have observed a $20 \%$ increase in the nearest-neighbor in-plane and out-of-plane matrix elements when going from LDA to $G W$. Comparision to ARPES and transport measurements suggest that LDA is not a good description of the electronic band structure of graphite because of the importance of correlation effects. We have explicitly shown that the accuracy of the TB- $G W$ parameters is sufficient to reproduce the QP band structure with an accuracy of $\sim 10 \mathrm{meV}$ for the higher energy points and $\sim 1 \mathrm{meV}$ for the bands that are relevant for electronic transport. Thus the $3 \mathrm{NN}$ TB- $G W$ calculated QP band energy dispersions are sufficiently accurate to be compared to a wide range of optical and transport experiments. The TB- $G W$ pa- rameters from Table I should be used in the future for interpretation of experiments that probe the band structure in graphite and FLG. For transport experiments that only involve electronic states close to the $K H$ axis (in graphite) or the $K$ point in FLG, the SWMC Hamiltonian with parameters from Table II can be used.

With the new set of TB parameters we have calculated the low-energy properties of graphite: the Fermi velocity, the Fermi surface, plasmon frequencies, and the ratio of conductivities parallel and perpendicular to the graphene layers and effective masses. These values are compared to experiments and we have demonstrated an excellent agreement with almost all values.

We have shown that at $\pm 25 \mathrm{meV}$, a semimetal to metal transition exists in graphite. Such a transition should be experimentally observable by HREELS or ARPES for electrondoped graphite single crystals with very low doping levels (i.e., the dilute limit). Experimentally, the synthesis of such samples is possible by evaporation of potassium onto the sample surface and choosing a sufficiently high equilibration temperature so that no staging compound forms. It would, thus, be interesting to do a combined ARPES and HREELS experiment on this kind of sample.

We have also discussed the issue of the existence of Dirac fermions in graphite and FLGs. For pristine graphite it is clear that for a finite value of the gap $\Delta$ at $H$ point, we 
always have a nonzero value of the effective masses at $H$. We now discuss some possibilities for $\Delta=0$. One way would be to use $A A$ stacked graphite. However, natural singlecrystal graphite has mainly $A B$ stacking, and the preparation of an $A A$-like surface by repeated cleaving is a rather difficult task. Recently, the possibility of reducing $\Delta$ by increasing the doping level has been put forward ${ }^{34,43}$ and this seems a more promising way for experiments. For the case of a fully doped graphite single crystal, the interlayer interaction is negligible because the distance between the graphene sheets is increased and the stacking sequence is changed to $A A$ stacking. These two effects would cause the appearance of Dirac fermions in fully doped graphite.

With the new set of 3 NN TB parameters we have calculated the QP dispersion of FLG in the whole 2D BZ. We have shown the evolution of the energy eigenvalue spectrum at $K$ point as a function of $N$, the number of layers. An interesting family pattern was observed, where the first-, second-, third-, etc. highest transitions form a pattern that approaches the energy-band width of bulk graphite with increasing $N$. For the highest transition, the bulk graphite band width is already reached at $N=15$. This family pattern is a direct result of the $A B$ stacking sequence in FLG and it can possibly be observed experimentally. It should be pointed out here that a very similar fine structure around the $K$ point in bulk graphite has been observed by optical absorption spectroscopy by Misu et al. ${ }^{49}$ although the family pattern from Fig. 13 might be observed also by ARPES on high-quality FLGs synthesized on $\mathrm{Ni}(111)$ (Ref. 3) or $\mathrm{SiC}$ (Ref. 2).

\section{ACKNOWLEDGMENTS}

A.G. acknowledges the Marie Curie Foundation (COMTRANS) from the European Union. A.R. and C.A. are supported in part by Spanish MEC (Grant No. FIS200765702-C02-01), Grupos Consolidados UPV/EHU of the Basque Country Government (Grant No. IT-319-07) European Community e-I3 ETSF and SANES (Grant No. NMP4CT-2006-017310) projects. L.W. acknowledges support from the French national research agency (Project PJC05_6741). R.S. acknowledges MEXT (Grants Nos. 20241023 and No. 16076201). We acknowledge M. Knupfer for critical reading of the manuscript. XCrysDen (Ref. 68) was used to prepare Fig. 11.

\section{APPENDIX}

We made computer programs to automatically generate the $3 \mathrm{NN}$ Hamiltonians and overlap matrices for $N$ graphene layers. The method is following the description by Partoens et $a l .{ }^{24}$ but with the difference that we include $3 \mathrm{NN}$ matrix elements that describe the QP dispersion in the whole BZ. It is interesting to note the similarity between the Hamiltonian of a $N$-layer graphene and a stage $N$-graphite intercalation compound. ${ }^{69}$ The general form of the Hamiltonian is given by

$$
H(\mathbf{k})=\left(\begin{array}{ccccc}
H_{A_{1} A_{1}} & H_{A_{1} B_{1}} & H_{A_{1} A_{2}} & \ldots & H_{A_{1} B_{N}} \\
H_{B_{1} A_{1}} & H_{B_{1} B_{1}} & H_{B_{1} A_{2}} & \ldots & H_{B_{2} B_{N}} \\
H_{A_{2} A_{1}} & H_{A_{2} B_{1}} & H_{A_{2} A_{2}} & \ldots & H_{A_{2} B_{N}} \\
\vdots & \vdots & \vdots & \vdots & \vdots \\
H_{B_{N} A_{1}} & H_{B_{N} B_{1}} & H_{B_{N} A_{2}} & \ldots & H_{B_{N} B_{N}}
\end{array}\right),
$$

and the overlap matrix is given by

$$
S(\mathbf{k})=\left(\begin{array}{ccccc}
S_{A_{1} A_{1}} & S_{A_{1} B_{1}} & S_{A_{1} A_{2}} & \ldots & S_{A_{1} B_{N}} \\
S_{B_{1} A_{1}} & S_{B_{1} B_{1}} & S_{B_{1} A_{2}} & \ldots & S_{B_{2} B_{N}} \\
S_{A_{2} A_{1}} & S_{A_{2} B_{1}} & S_{A_{2} A_{2}} & \ldots & S_{A_{2} B_{N}} \\
\vdots & \vdots & \vdots & \vdots & \vdots \\
S_{B_{N} A_{1}} & S_{B_{N} B_{1}} & S_{B_{N} A_{2}} & \ldots & S_{B_{N} B_{N}}
\end{array}\right) .
$$

The QP band structure is then given by solving equation Eq. (4). The 3NN TB Hamiltonians can be used with $G W$ parameters from Table I. In the following we give the explicit form for the $H(\mathbf{k})$ and $S(\mathbf{k})$ of a monolayer and an $A B$ stacked bilayer and $A B A$ trilayer which was used in Sec. VII.

\section{Monolayer graphene}

The monolayer 3NN Hamiltonian and overlap matrices are given by

$$
H(\mathbf{k})=\left(\begin{array}{cc}
E_{0}+\gamma_{0}^{2} f_{2}(\mathbf{k}) & \gamma_{0}^{1} f_{1}(\mathbf{k})+\gamma_{0}^{3} f_{3}(\mathbf{k}) \\
\gamma_{0}^{1} f_{1}(\mathbf{k})^{*}+\gamma_{0}^{3} f_{3}(\mathbf{k})^{*} & E_{0}+\gamma_{0}^{2} f_{2}(\mathbf{k})^{*}
\end{array}\right)
$$

and

$$
S(\mathbf{k})=\left(\begin{array}{cc}
1+f_{2}(\mathbf{k}) s_{0}^{2} & f_{1}(\mathbf{k}) s_{0}^{1}+f_{3}(\mathbf{k}) s_{0}^{3} \\
f_{1}(\mathbf{k})^{*} s_{0}^{1}+f_{3}(\mathbf{k})^{*} s_{0}^{3} & 1+f_{2}(\mathbf{k})^{*} s_{0}^{2}
\end{array}\right)
$$

\section{Bilayer graphene}

The bilayer 3NN Hamiltonian and overlap matrices are given by

$$
H(\mathbf{k})=\left(\begin{array}{cccc}
E_{0}+\Delta+\gamma_{0}^{2} f_{2}(\mathbf{k}) & \gamma_{0}^{1} f_{1}(\mathbf{k})+\gamma_{0}^{3} f_{3}(\mathbf{k}) & \gamma_{1} & \gamma_{4} f_{1}(\mathbf{k})^{*} \\
\gamma_{0}^{1} f_{1}(\mathbf{k})^{*}+\gamma_{0}^{3} f_{3}(\mathbf{k})^{*} & E_{0}+\gamma_{0}^{2} f_{2}(\mathbf{k})^{*} & \gamma_{4} f_{1}(\mathbf{k})^{*} & \gamma_{3} f_{1}(\mathbf{k}) \\
\gamma_{1} & \gamma_{4} f_{1}(\mathbf{k}) & E_{0}+\Delta+\gamma_{0}^{2} f_{2}(\mathbf{k}) & \gamma_{0}^{1} f_{1}(\mathbf{k})^{*}+\gamma_{0}^{3} f_{3}(\mathbf{k})^{*} \\
\gamma_{4} f_{1}(\mathbf{k}) & \gamma_{3} f_{1}(\mathbf{k})^{*} & \gamma_{0}^{1} f_{1}(\mathbf{k})+\gamma_{0}^{3} f_{3}(\mathbf{k}) & E_{0}+\gamma_{0}^{2} f_{2}(\mathbf{k})
\end{array}\right)
$$




$$
S(\mathbf{k})=\left(\begin{array}{cccc}
1+s_{0}^{2} f_{2}(\mathbf{k}) & s_{0}^{1} f_{1}(\mathbf{k})+s_{0}^{3} f_{3}(\mathbf{k}) & 0 & 0 \\
s_{0}^{1} f_{1}(\mathbf{k})^{*}+s_{0}^{3} f_{3}(\mathbf{k})^{*} & 1+s_{0}^{2} f_{2}(\mathbf{k})^{*} & 0 & 0 \\
0 & 0 & 1+s_{0}^{2} f_{2}(\mathbf{k}) & s_{0}^{1} f_{1}(\mathbf{k})^{*}+s_{0}^{3} f_{3}(\mathbf{k})^{*} \\
0 & 0 & s_{0}^{1} f_{1}(\mathbf{k})+s_{0}^{3} f_{3}(\mathbf{k}) & 1+s_{0}^{2} f_{2}(\mathbf{k})
\end{array}\right)
$$

\section{Trilayer graphene}

The trilayer Hamiltonian and overlap matrices read as

$$
H(\mathbf{k})=\left(\begin{array}{cccccc}
E_{0}+\Delta+\gamma_{0}^{2} f_{2}(\mathbf{k}) & f_{1}(\mathbf{k}) \gamma_{0}^{1}+\gamma_{0}^{3} f_{3}(\mathbf{k}) & \gamma_{1} & \gamma_{4} f_{1}(\mathbf{k})^{*} & \gamma_{5} & 0 \\
\gamma_{0}^{1} f_{1}(\mathbf{k})^{*}+\gamma_{0}^{3} f_{3}(\mathbf{k})^{*} & E_{0}+\gamma_{0}^{2} f_{2}(\mathbf{k})^{*} & \gamma_{4} f_{1}(\mathbf{k}) s & \gamma_{3} f_{1}(\mathbf{k}) & 0 & \gamma_{2} \\
\gamma_{1} & \gamma_{4} f_{1}(\mathbf{k}) & E_{0}+\Delta+\gamma_{0}^{2} f_{2}(\mathbf{k}) & \gamma_{0}^{1} f_{1}(\mathbf{k})^{*}+\gamma_{0}^{3} f_{3}(\mathbf{k})^{*} & \gamma_{1} & \gamma_{4} f_{1}(\mathbf{k}) \\
\gamma_{4} f_{1}(\mathbf{k}) & \gamma_{3} f_{1}(\mathbf{k})^{*} & \gamma_{0}^{1} f_{1}(\mathbf{k})+\gamma_{0}^{3} f_{3}(\mathbf{k}) & E_{0}+\gamma_{0}^{2} f_{2}(\mathbf{k})^{*} & \gamma_{4} f_{1}(\mathbf{k}) & \gamma_{3} f_{1}(\mathbf{k})^{*} \\
\gamma_{5} & 0 & \gamma_{1} & \gamma_{4} f_{1}(\mathbf{k})^{*} & E_{0}+\Delta+\gamma_{0}^{2} f_{2}(\mathbf{k}) & \gamma_{0}^{1} f_{1}(\mathbf{k})+\gamma_{0}^{3} f_{3}(\mathbf{k}) \\
0 & \gamma_{2} & \gamma_{4} f_{1}(\mathbf{k})^{*} & \gamma_{3} f_{1}(\mathbf{k}) & \gamma_{0}^{1} f_{1}(\mathbf{k})^{*}+\gamma_{0}^{3} f_{3}(\mathbf{k})^{*} & E_{0}+\gamma_{0}^{2} f_{2}(\mathbf{k})^{*}
\end{array}\right)
$$

and

$S(\mathbf{k})$

$$
=\left(\begin{array}{cccccc}
1+s_{0}^{2} f_{2}(\mathbf{k}) & f_{1}(\mathbf{k}) s_{0}^{1}+s_{0}^{3} f_{3}(\mathbf{k}) & 0 & 0 & 0 & 0 \\
s_{0}^{1} f_{1}(\mathbf{k})^{*}+s_{0}^{3} f_{3}(\mathbf{k})^{*} & 1+s_{0}^{2} f_{2}(\mathbf{k})^{*} & 0 & 0 & 0 & 0 \\
0 & 0 & 1+s_{0}^{2} f_{2}(\mathbf{k}) & s_{0}^{1} f_{1}(\mathbf{k})^{*}+s_{0}^{3} f_{3}(\mathbf{k})^{*} & 0 & 0 \\
0 & 0 & s_{0}^{1} f_{1}(\mathbf{k})+s_{0}^{3} f_{3}(\mathbf{k}) & 1+s_{0}^{2} f_{2}(\mathbf{k}) & 0 & 0 \\
0 & 0 & 0 & 0 & E_{0}+\Delta+s_{0}^{2} f_{2}(\mathbf{k})^{*} & s_{0}^{1} f_{1}(\mathbf{k})+s_{0}^{3} f_{3}(\mathbf{k}) \\
0 & 0 & 0 & 0 & s_{0}^{1} f_{1}(\mathbf{k})^{*}+s_{0}^{3} f_{3}(\mathbf{k})^{*} & 1+s_{0}^{2} f_{2}(\mathbf{k})^{*}
\end{array}\right)
$$

\section{Graphite}

For the case of three-dimensional graphite we have $\mathbf{k}=\left(k_{x}, k_{y}, k_{z}\right)$, and we use $\Gamma=2 \cos \left(k_{z} c_{0}\right)$ with $c_{0}=3.35 \AA$ (the distance between adjacent graphene planes). The Hamiltonian and overlap matrices of graphite are given by

$H(\mathbf{k})$

$$
=\left(\begin{array}{cccc}
E_{0}+\Delta+\gamma_{0}^{2} f_{2}(\mathbf{k})+\gamma_{5}\left(\Gamma^{2}-2\right) & \gamma_{0}^{1} f_{1}(\mathbf{k})+\gamma_{0}^{3} f_{3}(\mathbf{k}) & \Gamma \gamma_{1} & \Gamma \gamma_{4} f_{1}(\mathbf{k})^{*} \\
\gamma_{0}^{1} f_{1}(\mathbf{k})^{*}+\gamma_{0}^{3} f_{3}(\mathbf{k})^{*} & E_{0}+\gamma_{0}^{2} f_{2}(\mathbf{k})^{*}+\gamma_{2}\left(\Gamma^{2}-2\right) & \gamma_{4} f_{1}(\mathbf{k})^{*} \Gamma & \gamma_{3} \Gamma f_{1}(\mathbf{k}) \\
\gamma_{1} \Gamma & \gamma_{4} f_{1}(\mathbf{k}) \Gamma & E_{0}+\Delta+\gamma_{0}^{2} f_{2}(\mathbf{k})+\gamma_{5}\left(\Gamma^{2}-2\right) & \gamma_{0}^{1} f_{1}(\mathbf{k})^{*}+\gamma_{0}^{3} f_{3}(\mathbf{k})^{*} \\
\gamma_{4} f_{1}(\mathbf{k}) \Gamma & \gamma_{3} f_{1}(\mathbf{k})^{*} \Gamma & \gamma_{0}^{1} f_{1}(\mathbf{k})+\gamma_{0}^{3} f_{3}(\mathbf{k}) & E_{0}+\gamma_{0}^{2} f_{2}(\mathbf{k})+\gamma_{2}\left(\Gamma^{2}-2\right)
\end{array}\right)
$$

and

$$
S(\mathbf{k})=\left(\begin{array}{cccc}
1+s_{0}^{2} f_{2}(\mathbf{k}) & s_{0}^{1} f_{1}(\mathbf{k})+s_{0}^{3} f_{3}(\mathbf{k}) & 0 & 0 \\
s_{0}^{1} f_{1}(\mathbf{k})^{*}+s_{0}^{3} f_{3}(\mathbf{k})^{*} & 1+s_{0}^{2} f_{2}(\mathbf{k})^{*} & 0 & 0 \\
0 & 0 & 1+s_{0}^{2} f_{2}(\mathbf{k}) & s_{0}^{1} f_{1}(\mathbf{k})^{*}+s_{0}^{3} f_{3}(\mathbf{k})^{*}, \\
0 & 0 & s_{0}^{1} f_{1}(\mathbf{k})+s_{0}^{3} f_{3}(\mathbf{k}) & 1+s_{0}^{2} f_{2}(\mathbf{k})
\end{array}\right)
$$

respectively.

The sum of the phase factors for first-, second-, and third-nearest neighbors are given by $f_{1}(\mathbf{k}), f_{2}(\mathbf{k})$, and $f_{3}(\mathbf{k})$, respectively. Note that $f_{1}(\mathbf{k})$ and $f_{3}(\mathbf{k})$ couple $A$ and $B$ atoms and $f_{2}(\mathbf{k})$ describes the $A A$ and $B B$ interactions. The sum of phase factors are given by $f_{1}(\mathbf{k})=\exp \left(i k_{x} a_{0} / 2\right)+2 \exp \left(-i k_{x} a_{0} / 2\right) \cos \left(\sqrt{3} k_{y} a_{0} / 2\right), f_{2}(\mathbf{k})=\sum_{\ell=1}^{6} \exp \left(i \mathbf{k r} \mathbf{r}_{\ell}^{2}\right)$, and $f_{3}(\mathbf{k})=\Sigma_{\ell=1}^{3} \exp \left(i \mathbf{k} \mathbf{r}_{\ell}^{3}\right)$. Here $r_{\ell}^{2}$ and $r_{\ell}^{3}$ are the vectors that connect the $A_{1}$ atom (see Fig. 1) with the second-nearest $B$ atoms (six atoms) and the third-nearest $A$ atoms (three atoms), respectively. ${ }^{70}$ 
*Corresponding author. alexander.grueneis@univie.ac.at

${ }^{1}$ C. Berger, Z. Song, T. Li, X. Li, A. Y. Ogbazghi, R. Feng, Z. Dai, A. N. Marchenkov, E. H. Conrad, P. N. First, and W. A. de Heer, Science 312, 1191 (2006).

${ }^{2}$ Th. Seyller, K. V. Emtsev, K. Gao, F. Speck, L. Ley, A. Tadich, L. Broekman, J. D. Riley, R. C. G. Leckey, O. Rader, A. Varykhalov, and A. M. Shikin, Surf. Sci. 600, 3906 (2006).

${ }^{3}$ A. Grüneis and D. V. Vyalikh, Phys. Rev. B 77, 193401 (2008).

${ }^{4}$ K. S. Novoselov, A. K. Geim, S. V. Morozov, D. Jiang, Y. Zhang, S. V. Dubonos, I. V. Grigorieva, and A. A. Firsov, Science 306, 666 (2004).

${ }^{5}$ K. S. Novoselov, D. Jiang, F. Schedin, T. J. Booth, V. V. Khotkevich, S. V. Morozov, and A. K. Geim, Proc. Natl. Acad. Sci. U.S.A. 102, 10451 (2005).

${ }^{6}$ Li Xiaolin, Z. Guangyu, B. Xuedong, S. Xiaoming, W. Xinran, W. Enge, and H. Dai, Nat. Nanotechnol. 3, 538 (2008).

${ }^{7}$ A. Geim and K. Novoselov, Nature Mater. 6, 183 (2007).

${ }^{8}$ M. I. Katsnelson, K. S. Novoselov, and A. K. Geim, Nat. Phys. 2, 620 (2006).

${ }^{9}$ T. Ohta, A. Bostwick, T. Seyller, K. Horn, and E. Rotenberg, Science 313, 951 (2006).

${ }^{10}$ C. Berger, Z. Song, T. Li, X. Li, A. Y. Ogbazghi, R. Feng, Z. Dai, A. N. Marchenkov, E. H. Conrad, P. N. First, and W. A. de Heer, J. Phys. Chem. B 108, 19912 (2004).

${ }^{11}$ V. M. Karpan, G. Giovannetti, P. A. Khomyakov, M. Talanana, A. A. Starikov, M. Zwierzycki, J. van den Brink, G. Brocks, and P. J. Kelly, Phys. Rev. Lett. 99, 176602 (2007).

${ }^{12}$ A. Bostwick, T. Ohta, T. Seyller, K. Horn, and E. Rotenberg, Nat. Phys. 3, 36 (2007).

${ }^{13}$ A. Grüneis, C. Attaccalite, T. Pichler, V. Zabolotnyy, H. Shiozawa, S. L. Molodtsov, D. Inosov, A. Koitzsch, M. Knupfer, J. Schiessling, R. Follath, R. Weber, P. Rudolf, L. Wirtz, and A. Rubio, Phys. Rev. Lett. 100, 037601 (2008).

${ }^{14}$ S. Y. Zhou, G. H. Gweon, C. D. Spataru, J. Graf, D. H. Lee, S. G. Louie, and A. Lanzara, Phys. Rev. B 71, 161403(R) (2005).

${ }^{15}$ K. Sugawara, T. Sato, S. Souma, T. Takahashi, and H. Suematsu, Phys. Rev. Lett. 98, 036801 (2007).

${ }^{16}$ S. G. Louie, in Topics in Computational Materials Science, edited by C. Y. Fong (World Scientific, Singapore, 1997), p. 96.

${ }^{17}$ C. Heske, R. Treusch, F. J. Himpsel, S. Kakar, L. J. Terminello, H. J. Weyer, and E. L. Shirley, Phys. Rev. B 59, 4680 (1999).

${ }^{18}$ V. N. Strocov, A. Charrier, J. M. Themlin, M. Rohlfing, R. Claessen, N. Barrett, J. Avila, J. Sanchez, and M. C. Asensio, Phys. Rev. B 64, 075105 (2001).

${ }^{19}$ V. N. Strocov, P. Blaha, H. I. Starnberg, M. Rohlfing, R. Claessen, J.-M. Debever, and J.-M. Themlin, Phys. Rev. B 61, 4994 (2000).

${ }^{20}$ S. Reich, J. Maultzsch, C. Thomsen, and P. Ordejon, Phys. Rev. B 66, 035412 (2002).

${ }^{21}$ L. M. Malard, J. Nilsson, D. C. Elias, J. C. Brant, F. Plentz, E. S. Alves, A. H. Castro Neto, and M. A. Pimenta, Phys. Rev. B 76, 201401(R) (2007).

${ }^{22}$ A. Bostwick, T. Ohta, J. L. McChesney, T. Seyller, K. Horn, and E. Rotenberg, Solid State Commun. 143, 63 (2007).

${ }^{23}$ S. Y. Zhou, D. A. Siegel, A. V. Fedorov, F. El Gabaly, A. K. Schmid, A. H. Castro Neto, D.-H. Lee, and A. Lanzara, Nature Mater. 7, 259 (2008).

${ }^{24}$ B. Partoens and F. M. Peeters, Phys. Rev. B 74, 075404 (2006).

${ }^{25}$ K. Sasaki, J. Jiang, R. Saito, S. Onari, and Y. Tanaka, J. Phys. Soc. Jpn. 76, 033702 (2007).
${ }^{26}$ C. T. White, J. W. Li, D. Gunlycke, and J. W. Mintmire, Nano Lett. 7, 825 (2007)

${ }^{27}$ R. C. Tatar and S. Rabii, Phys. Rev. B 25, 4126 (1982); J. C. Slonczewski and P. R. Weiss, Phys. Rev. 109, 272 (1958).

${ }^{28}$ M. S. Dresselhaus and G. Dresselhaus, Adv. Phys. 30, 139 (1981).

${ }^{29}$ M. S. Dresselhaus and J. G. Mavroides, IBM J. Res. Dev. 8, 262 (1964).

${ }^{30}$ M. S. Dresselhaus, G. Dresselhaus, and J. E. Fischer, Phys. Rev. B 15, 3180 (1977).

${ }^{31}$ J. C. Charlier, X. Gonze, and J. P. Michenaud, Phys. Rev. B 43, 4579 (1991).

${ }^{32}$ S. Y. Zhou, G. H. Gweon, J. Graf, A. V. Fedorov, C. D. Spataru, R. D. Diehl, Y. Kopelevich, D. H. Lee, S. G. Louie, and A Lanzara, Nat. Phys. 2, 595 (2006).

${ }^{33}$ W. W. Toy, M. S. Dresselhaus, and G. Dresselhaus, Phys. Rev. B 15, 4077 (1977).

${ }^{34}$ C. Attaccalite, A. Grüneis, T. Pichler, and A. Rubio, arXiv:0808.0786 (unpublished).

${ }^{35}$ X. Gonze, J. M. Beuken, R. Caracas, F. Detraux, M. Fuchs, G. M. Rignanese, L. Sindic, M. Verstraete, G. Zerah, F. Jollet, M. Torrent, A. Roy, M. Mikami, Ph. Ghosez, J. Y. Raty, and D. C. Allan, Comput. Mater. Sci. 25, 478 (2002).

${ }^{36}$ M. S. Hybertsen and S. G. Louie, Phys. Rev. B 34, 5390 (1986).

${ }^{37}$ L. Hedin, Phys. Rev. 139, A796 (1965).

${ }^{38}$ A. Marini, the Yambo project, http://www.yambo-code.org/.

${ }^{39}$ G. P. Mikitik and Yu. V. Sharlai, Phys. Rev. B 73, 235112 (2006).

${ }^{40}$ D. Gunlycke and C. T. White, Phys. Rev. B 77, 115116 (2008).

${ }^{41}$ H. Venghaus, Phys. Status Solidi B 71, 609 (1975).

${ }^{42}$ E. T. Jensen, R. E. Palmer, W. Allison, and J. F. Annett, Phys. Rev. Lett. 66, 492 (1991).

${ }^{43}$ A. Grüneis, C. Attaccalite, A. Rubio, D. Vyalikh, S. L. Molodtsov, J. Fink, R. Follath, W. Eberhardt, B. Büchner, and T. Pichler, arXiv:0808.1613 (unpublished).

${ }^{44}$ A. Grüneis, C. Attaccalite, A. Rubio, S. L. Molodtsov, D. V. Vyalikh, J. Fink, R. Follath, and T. Pichler, Phys. Status Solidi B 245, 2072 (2008).

${ }^{45}$ S. B. Trickey, F. Müller-Plathe, G. H. F. Diercksen, and J. C. Boettger, Phys. Rev. B 45, 4460 (1992).

${ }^{46}$ Edward McCann and Vladimir I. Falko, Phys. Rev. Lett. 96, 086805 (2006).

${ }^{47}$ Sylvain Latil and Luc Henrard, Phys. Rev. Lett. 97, 036803 (2006).

${ }^{48}$ Mikito Koshino and Tsuneya Ando, Physica E (Amsterdam) 40, 1014 (2008).

${ }^{49}$ A. Misu, E. Mendez, and M. S. Dresselhaus, J. Phys. Soc. Jpn. 47, 199 (1979).

${ }^{50}$ M. Lazzeri, C. Attaccalite, L. Wirtz, and F. Mauri, Phys. Rev. B 78, 081406(R) (2008).

${ }^{51}$ A. Grüneis, T. Pichler, H. Shiozawa, C. Attaccalite, L. Wirtz, S. L. Molodtsov, R. Follath, R. Weber, and A. Rubio, Phys. Status Solidi B 244, 4129 (2007).

${ }^{52} \mathrm{~A}$. Grüneis et al. (unpublished).

${ }^{53}$ J. Maultzsch, S. Reich, C. Thomsen, H. Requardt, and P. Ordejon, Phys. Rev. Lett. 92, 075501 (2004).

${ }^{54}$ E. Mendez, A. Misu, and M. S. Dresselhaus, Phys. Rev. B 21, 827 (1980).

${ }^{55}$ J. M. Zhang and P. Eklund, J. Mater. Res. 2, 858 (1987).

${ }^{56}$ J. Geiger, H. Katterwe, and B. Schröder, Z. Phys. 241, 45 
(1971).

${ }^{57}$ L. Vitali, M. A. Schneider, K. Kern, L. Wirtz, and A. Rubio, Phys. Rev. B 69, 121414(R) (2004).

${ }^{58}$ P. Laitenberger and R. E. Palmer, Phys. Rev. Lett. 76, 1952 (1996).

${ }^{59}$ M. Zanini and J. Fischer, Mater. Sci. Eng. 31, 169 (1977).

${ }^{60}$ D. T. Morelli and C. Uher, Phys. Rev. B 30, 1080 (1984).

${ }^{61} \mathrm{G}$. Li and E. Andrei, Nat. Phys. 3, 623 (2007).

${ }^{62}$ M. Orlita, C. Faugeras, G. Martinez, D. K. Maude, M. L. Sadowski, and M. Potemski, Phys. Rev. Lett. 100, 136403 (2008).

${ }^{63}$ K. Sugawara, T. Sato, S. Souma, T. Takahashi, and H. Suematsu, Phys. Rev. B 73, 045124 (2006).

${ }^{64}$ A. R. Law, M. T. Johnson, and H. P. Hughes, Phys. Rev. B 34,
4289 (1986).

${ }^{65}$ E. Mendez, T. C. Chieu, N. Kambe, and M. S. Dresselhaus, Solid State Commun. 33, 837 (1980).

${ }^{66}$ J. K. Galt, W. A. Yager, and H. W. Dail, Phys. Rev. 103, 1586 (1956).

${ }^{67}$ D. E. Soule, Phys. Rev. 112, 698 (1958).

${ }^{68}$ A. Kokalj, Comput. Mater. Sci. 28, 155 (2003).

${ }^{69}$ R. Saito and H. Kamimura, Phys. Rev. B 33, 7218 (1986).

${ }^{70} \mathrm{~A}$ Mathematica program for calculation of the electron energy band structure with the new TB- $G W$ set of parameters is available for download at http://homepage.univie.ac.at/ alexander.grueneis/. 